\title{
2.3 Diskussion
}

In dieser Arbeit wurde in Anknüpfung an zwei bekannte kognitive Interferenzhypothesen die Frage untersucht, ob gewalthaltige Medien Gedächtnisfunktionen über die kurzfristige Vermittlung von Distress negativ beeinflussen können. Dabei wurden Fragestellungen untersucht, die sowohl emotionale als auch kognitive Wirkungen der Medienrezeption umfassen:

1. Welche Faktoren tragen dazu bei, dass bei der Rezeption gewalthaltiger Medien in besonderem Maße Distress erlebt wird?

2. Welchen Einfluss hat die Rezeption von gewalthaltigen Bildschirmmedien auf die Konsolidierung von Lerninhalten?

3. Welchen Einfluss hat die Rezeption von gewalthaltigen Bildschirmmedien auf die Arbeitsgedächtnisleistung?

4. Inwieweit weisen gewalthaltige Computerspiele und gewalthaltige Filme hinsichtlich des emotionalen und kognitiven Wirkpotentials eine differentielle Bedeutung auf?

\subsubsection{Diskussion des emotionalen Wirkpotentials gewalthaltiger Bildschirmmedien}

Die Überprüfung der emotionalen Wirkhypothesen ergab, dass die Versuchspersonen erwartungskonform mit höherem Distress auf die gewalthaltigen Medien als auf die gewaltneutralen Angebote reagierten. Dieser Befund steht in Übereinstimmung mit nahezu allen anderen zu diesem Gegenstandsbereich veröffentlichten empirischen Untersuchungen (vgl. Kapitel 1.3.3.1). So konnten in einer Vielzahl von Experimenten erhöhte kardiovaskuläre Reaktionen, katecholamine Stressreaktionen und ein subjektiv erhöhtes Distresserleben in Folge der Nutzung gewalthaltiger gegenüber gewaltneutraler Computerspiele ermittelt werden (vgl. Tabelle 3). Der weit überwiegende Teil dieser Studien wurde mit erwachsenen Versuchspersonen durchgeführt, die hinsichtlich ihres Alters mit der vorliegenden Untersuchungsstichprobe vergleichbar sind. Damit kann mit den vorliegenden Daten die grundsätzliche Annahme bestätigt werden, dass die Nutzung gewalthaltiger Medien auch über das Kindes- und Jugendalter hinaus Distresserleben auslösen kann. Hierin bestätigt sich die Vermutung, dass Arousalwirkungen von gewalthaltigen Spielen nicht ausschließlich als valenzneutrale Erregungssteigerung zu interpretieren sind, sondern auch ein negatives Gefühlserleben auf Seiten des Rezipienten kennzeichnen können.

Die Befunde weisen hierbei jedoch auf ein moderates Distresserleben der meisten Versuchsteilnehmer hin, indem der Gesamtskalenwert in den gewalthaltigen 
Bedingungen zwar höher ausfällt als in den gewaltneutralen, hierbei jedoch einen Durchschnittswert von 7 bis 8 von insgesamt 27 möglichen Punkten erreicht (vgl. Kapitel 2.2.4.1; Tabelle 22). Somit waren offenbar starke Gefühle von Stress oder Ekel und insbesondere Angst bei der Nutzung der berücksichtigten gewalthaltigen Medien zumindest nicht die Regel und nur wenige Versuchspersonen gaben an, durchgängig negative Gefühle in Konfrontation mit dem gewalthaltigen Stimulusmaterial erleben zu haben (vgl. Abbildung 28). Dieser Befund erscheint insbesondere vor dem Hintergrund bemerkenswert, dass in der vorliegenden Untersuchung mit vergleichsweise "harten" Gewaltkriterien gearbeitet wurde, indem die eingesetzten Medien ernsthafte physische Schädigungen von Menschen oder menschenähnlicher Wesen behandeln und durch eine explizite und naturalistische Darstellung der Gewaltfolgen gekennzeichnet sind (vgl. Kapitel 2.1.2.1). Die Intensität des eingesetzten Stimulusmaterials wird im Manipulationscheck grundsätzlich bestätigt, indem die gewalthaltigen Filme und Spiele von den Versuchspersonen mit rund acht von zehn Punkten als sehr gewalthaltig eingestuft wurden (vgl. Kapitel 2.2.3). Hieraus kann die Schlussfolgerung abgeleitet werden, dass die Versuchspersonen zwar die objektive Reizintensität der gewalthaltigen Medien als solche erkannten, ihr Gefühlsleben jedoch nicht in gleicher Weise beeinflusst wurde. Zwar wurde vorab ein höheres Distresserleben in Reaktion auf die präsentierte Mediengewalt erwartet, der Befund steht jedoch in Übereinstimmung mit grundsätzlichen Überlegungen, die mit der Wahl einer erwachsenen Stichprobe verbunden waren (vgl. Kapitel 2.1.1). So kann bei erwachsenen Personen aufgrund der Häufigkeit ihrer bisherigen Unterhaltungsmediennutzung (vgl. Kapitel 1.1.1) und dem hohen Anteil von Gewalt in den Medien (vgl. Kapitel 1.1.2) unterstellt werden, dass die meisten dieser Personen im Lauf ihres Lebens Copingstrategien im Umgang mit entsprechenden Angeboten entwickeln konnten und dass bei ihnen zudem bereits eine gewisse Gewöhnung an die Gewalt eingetreten ist. Aus der impliziten Annahme, dass Gewaltmedien im Erwachsenenalter bei den meisten Personen (im Gegensatz zum Kindesalter) keine gravierenden emotionalen Wirkungen mehr befürchten lassen, bezieht letztlich die Alterseinstufungspraxis von Unterhaltungsmedien und damit ein zentraler Eckpfeiler des Jugendmedienschutzsystems eine wesentliche Legitimationsgrundlage.

Die mit der Wahl einer erwachsenen Stichprobe verbundenen Einschränkungen waren bei der Studienkonzeption bekannt, weshalb subjektiven Stressmoderatorvariablen im Rahmen der Untersuchungsplanung ein großer Stellenwert beigemessen wurde. Hiermit sollten differenzierte Aussagen zu Versuchspersonen ermöglicht werden, bei denen trotz eines höheren Lebensalters erwartet werden kann, dass sie besonders stark auf gewalthaltige Medien reagieren. Die Frage, welche Variablen hier eine maßgebliche Rolle spielen, stand bislang jedoch kaum im Fokus der Forschung (vgl. Kapitel 1.4.4.2). In der vorliegenden Untersuchung konnte 
gezeigt werden, dass erhöhtem Neurotizismus und geringer Selbstwirksamkeitserwartung, die als Prädiktoren erhöhter Reagibilität in Konfrontation mit anerkannten psychosozialen Stressparadigmen diskutiert werden (vgl. Kirschbaum, et al., 1995), keine Bedeutung für die Vorhersage von Distresserleben bei der Nutzung gewalthaltiger Medien zukommt (vgl. Kapitel 2.2.4.1). Sehr wohl zeigt sich aber, dass ein erhöhtes $\mathrm{Ma} ß$ an prosozialen Einstellungen, geringe Erfahrungen mit Gewaltmedien in den letzten sechs Monaten und eine geringe Vorliebe für gewalthaltige Angebote ein erhöhtes rezeptionsbedingtes Distresserleben vorhersagen und hierbei nahezu 40 Prozent der interindividuellen Variabilität des Distresserlebens erklären. Diese Befunde knüpften unter anderem an die Desensibilisierungsforschung an. So konnten Bartholow und Kollegen (2006) zeigen, dass Personen mit hoher habitueller Gewaltspielexposition mit nach EEG-Bild geringeren Erregungsreaktionen auf gewalthaltige Bilder reagieren als Probanden mit geringer Gewaltspielexposition (vgl. Kapitel 1.4.4.2). Somit zeigt sich anhand der vorliegenden Daten, dass hiervon nicht nur ein physiologisches Erregungsmaß sondern auch subjektives Distresserleben betroffen ist. Dass Gewaltpräferenz Distresserleben bei der Nutzung gewalthaltiger Medien vorhersagen kann, und hierbei den stärksten der untersuchten Prädiktoren darstellt, erscheint vor diesem Hintergrund nur allzu plausibel. So wünschen sich Personen vermutlich gerade dann einen geringen Grad von Gewaltdarstellungen in actionreichen Unterhaltungsformaten, wenn sie auf diese Darstellungsmittel besonders empfindlich reagieren. Insbesondere die Bedeutung des Persönlichkeitsmerkmals Prosozialität als Prädiktor von Distresserleben ergänzt die bestehende Datenlage und knüpft an Untersuchungen an, die Zusammenhänge zwischen geringer Prosozialität und Gewaltmedienrezeption nachweisen konnte (vgl. Funk, Baldacci, Pasold, \& Baumgardner, 2004; Mößle, et al., 2007). Die Annahme, dass Gewaltmediennutzung prosoziale Einstellungen reduzieren kann, ist auch maßgeblicher Bestandteil der langfristigen Wirkkomponente des General Aggression Models (vgl. Kapitel 1.3.3). Die vorliegende Untersuchung zeigt, dass prosoziale Einstellungen für eine genussvolle Rezeption von Mediengewalt offenbar eher "hinderlich" sind, ein Befund, der innerhalb eines größeren Interpretationskontextes zum einen erklären kann, warum sich gerade Personen mit geringer Prosozialität besonders stark zu gewalthaltigen Medien hingezogen fühlen, zum anderen aber auch, warum bei langfristiger Gewaltmedienrezeption eine Anpassung von Norm- und Wertüberzeugungen und damit eine Verringerung von Prosozialität eintreten könnte. Derartige Veränderungen normativer Einstellungen und Überzeugungen könnten als funktionale Anpassungen verstanden werden, die dabei helfen, die mit der Gewaltmedienrezeption auftretenden negativen Gefühle zunehmend zu reduzieren.

Auch hinsichtlich geschlechtsbedingter Unterschiede im gewaltmedienrezeptivbedingten Distresserleben liefert die vorliegende Untersuchung einige Ergän- 
zungen und Anknüpfungen zum bestehenden Forschungsstand. Das weibliche Personen oftmals in höherem Maße mit negativen Gefühlen auf fiktionale Gewaltschilderungen reagieren ist bereits an anderer Stelle berichtet worden (vgl. Cantor, 2001; van der Molen \& Bushman, 2008). Es bestand bislang jedoch noch keine hinlängliche Kenntnis darüber, wie dieser Geschlechtsunterschied zu erklären ist. Cantor (2001) formulierte die Vermutung, dass ein stärkeres Antworten nach sozialer Erwünschtheit bei männlichen Personen ursächlich sein könnte, indem männliche Personen sich in Selbstauskünften gerne als vermeintlich männlich/hart darstellen und es deshalb eher nicht eingestehen, wenn sie in Konfrontation mit gewalthaltigen Medien Ängste oder andere negative Gefühle erleben. Mit der vorliegenden Untersuchung kann als alternative Erklärung angeboten werden, dass weibliche Personen in höherem Maße prosoziale Einstellungen internalisiert haben, Mediengewalt stärker ablehnen und auch geringere Erfahrungen damit aufweisen und aus diesen Gründen stärker mit negativen Gefühlen reagieren, wenn sie mit derartigen Angeboten konfrontiert werden. Damit tragen die Ergebnisse zu den subjektiven Stressmoderatorvariablen insgesamt zu einem besseren Verständnis dafür bei, warum unterschiedliche Personen auch unterschiedlich stark auf gewalthaltige Medien reagieren.

Weiterhin sollte in dem Experiment die aktuelle Forschungsfrage untersucht werden, ob sich gewalthaltige Computerspiele und Filme hinsichtlich ihres emotionalen Wirkpotentials voneinander unterscheiden. Die einzige zu dieser Frage aufgefundene Referenzstudie von Maass und Kollegen (2010) erbrachte gemischte Befunde, indem kardiovaskuläre Indikatoren auf ein höheres Aktivierungspotential der gewalthaltigen Spiel- gegenüber der Filmbedingung hindeuteten, während stresshormonelle Parameter sowie die Selbsteinschätzung des Distresserlebens zwischen den Medienbedingungen nicht divergierte. Aus bestehenden Erkenntnissen zu der vergleichsweise hohen Ereignisfrequenz von Gewalthandlungen in gewalthaltigen Computerspielen (vgl. Kapitel 1.1.2) sowie der kognitiven Beanspruchung und dem Handlungsdruck in actionorientierten Computerspielen (vgl. Kapitel 1.3.1) wurde für die vorliegende Untersuchung die Hypothese abgeleitet, dass gewalthaltige Computerspiele eine höhere objektive Reizintensität aufweisen sollten, als gewalthaltige Filme vergleichbarer Genrekategorien, und dass diese damit auch ein erhöhtes Distresserleben bedingen müssten. Diese Annahme konnte jedoch nicht bestätigt werden (vgl. Kapitel 2.2.4.1): Beide Gewaltmedien unterschieden sich nicht signifikant in Hinblick auf ihr emotionales Wirkpotential. Aufgrund der in der vorliegenden Untersuchung zur Anwendung gebrachten engen Auswahlkriterien hinsichtlich des Gewaltgehalts der einbezogenen Medien (vgl. Kapitel 2.1.2.1) sowie durch die Realisierung von drei unterschiedlichen Angeboten in jeder Gewaltbedingung, die zudem hinsichtlich der berücksichtigten Genrekategorie (Action, Martial Arts, Horror) unmittelbar aufeinander bezogen wur- 
den (vgl. Kapitel 2.1.3), kann dieser Befund zumindest nicht durch eine hochselektiven Stimulusauswahl erklärt werden. Stattdessen kann als mögliche Begründung angeboten werden, dass gewalthaltige Computerspiele zwar in der Regel eine weit größere Anzahl an Gewalthandlungen aufweisen als Filme vergleichbarer Genrekategorien, die Darstellung dieser Gewaltereignisse jedoch trotz der fortgeschrittenen Technik noch nicht den Detaillierungs- und Realitätsgrad filmischer Darstellungen erreicht (vgl. Anhang E). Diese Annahme wird durch die Daten zum subjektiven Distresserleben insofern deskriptiv bestätigt, dass die Probanden der Gewaltspielbedingung in geringerem Maße Angst und Ekel erlebt haben als die Versuchspersonen der Gewaltfilmbedingung (vgl. Tabelle 22) und im Trend sogar geringerer Distress in der gewalthaltigen Spielbedingung erlebt wurde. Auf der anderen Seite wurde die Dimension "Stress" besonders häufig in der Gewaltspielbedingung benannt. Dies könnte darauf hinweisen, dass gerade bei Computerspielen als interaktivem Medium eine allgemeine Komponente des Handlungsdrucks, welcher sich primär auf die mit der Lösung der spielbezogenen Aufgaben und Anforderungen bezieht, von einer Komponente des negativen Affekterlebens abgegrenzt werden sollte, die sich primär aus der Reizintensität der Gewaltdarstellungen und der Bedrohlichkeit des Spielszenarios ergeben könnte. Diese Überlegung, die mit Hilfe der vorliegenden Arbeit jedoch nicht belegt werden kann, gibt Hinweise darauf, dass emotionale Reaktionen auf gewalthaltige Computerspiele zukünftig noch differenzierter erfasst und hierfür geeignete Messinstrumente entwickelt werden sollten.

\subsubsection{Diskussion des kognitiven Wirkpotentials gewalthaltiger Bildschirmmedien}

Im Fokus der vorliegenden Untersuchung stand die Überprüfung der Wirkungen gewalthaltiger Bildschirmmedien auf kognitive Informationsverarbeitungsprozesse. Entgegen der Erwartungen konnten keine negativen Wirkungen gewalthaltiger Medienangebote auf die untersuchten kognitiven Leistungsparameter aufgefunden werden. Dieser Befund zeigt sich unter Berücksichtigung der Art des genutzten Mediums und dem Vorhandensein erhöhter oder geringer Gewaltpräferenz auf Seiten des Rezipienten.

Zu der Frage, wie sich Gewaltmediennutzung auf die Konsolidierung von Lerninhalten auswirken kann, lagen zum Zeitpunkt der Konzeption und Durchführung dieser Untersuchung keine veröffentlichten empirischen Daten vor. Zwischenzeitlich konnten Maas und Kollegen (2009) im Rahmen eines Experimentes mit erwachsenen Personen im Alter von rund 22 Jahren, in welcher sowohl gewalthaltige als auch gewaltneutrale Filme und Spiele berücksichtigt wurden, keinen signifikanten Effekt der Treatmentbedingungen auf die verbale Gedächtnisleistung nach- 
weisen (vgl. Kapitel 1.3.2). Eine weitere publizierte Studie zur Gedächtnis-DefizitHypothese wurde mit Kindern durchgeführt und ermittelte im Gegensatz zu der Studie von Maass und Kollegen (2009) eine beeinträchtigende Wirkung auf die verbale Gedächtnisleistung durch emotionalisierende Medieninhalte (Dworak, et al., 2007). Dworak und Kollegen operationalisierten Gedächtnisleistung dabei in derselben Weise wie in der vorliegenden Untersuchung mittels des Visuellen und Verbalen Merkfähigkeitstest (VVM). Ob die Abweichung im Befund durch das geringere Lebensalter der Versuchspersonen oder durch methodische Mängel, insbesondere die auch innerhalb eines Within-Subjects-Designs mit $\mathrm{N}=11$ als sehr klein zu bewertende Stichprobe (vgl. Kapitel 1.3.2), erklärt werden kann, ist an dieser Stelle nicht eindeutig zu beantworten. In der vorliegenden Arbeit weisen Versuchspersonen der gewaltneutralen oder gewalthaltigen Medienbedingungen keine Unterschiede hinsichtlich ihrer räumlichen und visuellen Gedächtnisleistung auf. Im verwendeten Lerntestverfahren zeigt sich sogar entgegen der formulierten Hypothese, dass die Versuchspersonen der Gewaltbedingungen am zweiten Tag über ein signifikant höheres Vokabular der fiktiven Fremdsprache verfügen als die Personen der Gewaltneutralbedingungen (vgl. Abbildung 39). Wie kann dieses Resultat erklärt werden?

Insgesamt deckt sich der Befund mit dem Ergebnis, dass die Versuchspersonen nur ein geringes bis moderates Distresserleben in den Gewaltbedingungen erlebten. Hieraus kann die Vermutung abgeleitet werden, dass das Distresserleben nicht nur zu gering war, um kognitive Leistungseinbußen verursachen zu können, sondern insgesamt vielmehr im Sinne eines leicht erhöhten Arousals zu einer Stimulierung des Lernens beigetragen haben könnte. Wie ausgeführt wurde, wird von moderaten Arousalzuständen angenommen, dass sie kognitive Prozesse entweder gar nicht beeinflussen oder aber sie eher fördern denn beeinträchtigen (vgl. die Ausführungen zum U-funktionalen Zusammenhang von Stress und Kognition in Kapitel 1.4.3). Dies gilt insbesondere für die Konsolidierung, für die ein hinreichender Schweregrad von Stress als erforderlich gelten kann, um die Festigung der bereits initial gelernten Informationen als Post-Learning-Reiz zu beeinträchtigen. Warum sich jedoch dieser Befund nur im Lerntestverfahren, nicht aber im Gedächtnistest zeigt, kann nicht zweifelsfrei geklärt werden. Möglicherweise spielt hier die größere Sensitivität des Lerntests WERNICKO für situative Schwankungen der Lernleistung eine Rolle (vgl. Kapitel 2.1.4.2), während dem Gedächtnistest ein stärkeres Trait-Verständnis zugrundeliegt, welches dieses gegenüber situativen Einflüssen robuster macht (vgl. Kapitel 2.1.4.1). Zumindest in Hinblick auf Lernprozesse im Erwachsenenalter steht der vorliegende Befund, dass Konsolidierungsvorgänge durch gewalthaltige Medien nicht negativ beeinflusst werden, nicht nur in Übereinstimmung mit der Studie von Maass und Kollegen (2009) sondern auch mit einer weiteren zwischenzeitlich durchgeführten, jedoch bislang nicht publizierten Un- 
tersuchung. So konnten in einer Kooperationsstudie, die von der Klinik für Neurologie der Otto-von-Guericke Universität Magdeburg durchgeführt wurde (zum Studiendesign vgl. Pfeiffer et. al., 2006), ebenfalls keine kurzfristigen negativen Auswirkungen der Rezeption gewalthaltiger Filme auf die Konsolidierung expliziter Lerninhalte aufgefunden werden.

In der vorliegenden Untersuchung wurde zusätzlich mittels eines Konzentrationsleistungstests der Einfluss gewalthaltiger Medien auf die Weiterverarbeitung von Informationen sowie die damit in Zusammenhang stehenden Kontrollanforderungen erfasst (vgl. Kapitel 2.1.4.3). Zu der Frage, wie sich gewalthaltige Computerspiele auf die Konzentrationsleistung auswirken, liegen bislang nur lückenhafte Forschungsdaten vor (vgl. Kapitel 1.3.2). Boot und Kollegen (2008) konnten in Folge eines mehrwöchigen Computerspieltrainings mit verschiedenen gewaltneutralen und gewalthaltigen Angeboten keinerlei Veränderungen arbeitsgedächtnisnaher kognitiver Leistungen nachweisen. In der Untersuchung von Skosnik und Kollegen (2000) zeigte sich, dass Versuchspersonen nach der Nutzung eines gewalthaltigen Computerspiels zwar schneller auf Zielreize reagieren konnten, gleichzeitig aber in geringerem Maße zur Ausblendung irrelevanter Störreize in der Lage waren. Bei Reinecke und Trepte (2008) ergab sich unter Einsatz des Konzentrations-Leistungs-Tests, der auch in der vorliegenden Untersuchung eingesetzt wurde, dass Probanden nach der kurzen Nutzung eines gewaltneutralen Computerspiels über eine höhere Konzentrationsleistung verfügten als Probanden, die sich im Rahmen des Treatments selbstselektiv keinem Computerspiel zugewendet hatten. In der vorliegenden Studie wurden die Probanden hingegen randomisiert den Treatmentbedingungen zugewiesen. Hier konnten weder leistungsförderliche noch leistungsbeeinträchtigende Effekte der Nutzung gewalthaltiger Medien im Allgemeinen und gewalthaltiger Computerspiele im Speziellen aufgefunden werden. Dies kann insofern überraschen, als dass komplexe arbeitsgedächtnisintensive Leistungen als besonders störanfällig gelten und in der Regel bereits unter moderater Arousalerhöhung weniger effizient ausgeführt werden (vgl. Kapitel 1.4.3.). Inwieweit dieser Befund weitere Zweifel an der Annahme rechtfertigt, dass gewalthaltige Medien im Erwachsenenalter noch intensive Stressreaktionen (im Sinne einer Aktivierung der HPA-Achse) auslösen können, muss vor dem Hintergrund der in dieser Studie erhobenen Daten jedoch offen bleiben.

Insgesamt kann die Falsifikation der in der vorliegenden Untersuchung postulierten kognitiven Wirkhypothesen als stark gelten. Hier ist zum einen erneut auf die Intensität der einbezogenen Gewaltangebote hinzuweisen, zum anderen auf die mit 3 x 20-minütigen Treatments vergleichsweise hohe Stimulusdimensionierung (vgl. Kapitel 2.1.3). Es erscheint somit kaum die Annahme vertretbar, dass sich kognitive Wirkungen bei noch längeren Treatmentphasen gezeigt hätten, eine Vermutung, die bei einer sehr kurzen Stimulusdauer durchaus hätte naheliegen können. 
Die Post-Hoc-Poweranalysen weisen auf eine hohe Teststärke der eingesetzten Verfahren hin (vgl. Kapitel 2.2.4.2.3; Anhang J). Zudem wurde eine sehr heterogene und auch große Stichprobe von jungen Frauen und Männer unterschiedlicher Bildungsgrade und Computerspielerfahrungen einbezogen. Somit beschränkt sich der Befund auch nicht auf eine sehr homogene Gruppe von Versuchspersonen.

Ergänzend wurde im Rahmen explorativer Analysen untersucht, ob biographisch zurückliegende sowie aktuelle Mediennutzungsparameter mit kognitiven Leistungen im Erwachsenenalter assoziiert sind. Die Daten liefern hier einige ergänzende Anknüpfungspunkte an bereits bestehende Erkenntnisse. So zeigt sich unter Kontrolle des elterlichen Bildungshintergrunds und der Eltern-Kind-Beziehung, dass bei männlichen im Gegensatz zu weiblichen Versuchspersonen negative Zusammenhänge zwischen der frühkindlichen sowie kindlichen Fernsehnutzung und der verbalen Intelligenz im Erwachsenenalter bestehen (vgl. Tabelle 34). Dieser Befund steht zumindest in Bezug auf die Männer in Einklang mit der Forschungslage (vgl. Kapitel 1.2.2.1). So wurde in vielfältigen Querschnitt- und Längsschnittuntersuchungen auf negative Zusammenhänge zwischen der Fernsehnutzungszeit und der Entwicklung sprachbezogener Fähigkeiten, insbesondere der Lesefertigkeit, hingewiesen (vgl. die in Kapitel 1.2.2.1 diskutierten Studien; Neumann, 1988; Comstock \& Scharrer, 1999; Koolstra, et al., 1997; Ennemoser \& Schneider, 2007; Gortmaker, et al., 1990; Zimmermann \& Christakis, 2005). Dabei gilt insbesondere die Unterhaltungsfernsehzeit als problematisch, nicht jedoch die Nutzung edukativer Inhalte (Koolstra, et al., 1997; Ennemoser \& Schneider, 2007). Hierin könnte eine mögliche Erklärung dafür gesehen werden, warum in der vorliegenden Untersuchung die zurückliegende Fernsehnutzungszeit bei Frauen nicht mit der verbalen Intelligenz in Zusammenhang steht. Wie aufgezeigt werden konnte, geht eine höhere Mediennutzungszeit gerade bei Jungen häufig mit der Nutzung gewaltbetonter Inhalte einher (vgl. Kapitel 1.1.2). Zudem weisen Jungen schon in frühem Lebensalter eine hohe Fernsehnutzungszeit auf (vgl. Kapitel 1.1.1), wobei im Allgemeinen gilt, dass höhere Mediennutzungszeiten eine häufigere Nutzung unterhaltungsorientierter Angebote anzeigen (vgl. Kapitel 1.1.2). Somit könnte die Annahme abgeleitet werden, dass bei weiblichen Personen deshalb keine Zusammenhänge zwischen kindlicher Fernsehnutzungszeit und verbaler Intelligenz im Erwachsenenalter aufgefunden werden konnten, weil beeinträchtigende und stimulierende Aspekte der Mediennutzung bei dieser Gruppe in einem ausgewogeneren Verhältnis zueinander stehen. Bei männlichen Personen könnte die reine Fernsehzeit hingegen akkurater die Unterhaltungsfernsehzeit sowie die Nutzung gewalthaltiger Inhalte abbilden, weshalb diese auch ohne differenzierte Betrachtung der Inhalte negativ mit späterer verbaler Intelligenz korreliert. Diese Vermutung kann jedoch, da die Nutzungsinhalte der Medienbiographie in der vorliegenden Untersuchung nicht erfasst wurde (vgl. Kapitel 2.2.5.1), anhand der Daten nur 
indirekt abgeleitet werden. Insgesamt stehen die vorliegenden querschnittlichen Analysen jedoch in Abweichung zu Befunde, die längsschnittlich auf Zusammenhänge von Fernsehnutzung und einer späteren Beeinträchtigung von Gedächtnisfunktionen hinweisen (vgl. Zimmermann \& Christakis, 2005).

Hinsichtlich der Computerspielnutzungsbiographie zeigen sich ausschließlich bei weiblichen Versuchspersonen negative Zusammenhänge mit Konzentrationsleistung und Lernleistung im Erwachsenenalter (vgl. Tabelle 33). Bei männlichen Versuchspersonen steht Computerspielnutzung im Kindes- und Jugendalter hingegen mit besserer Konzentrationsleistung in Zusammenhang. Wie kann dieses gegenläufige Befundmuster erklärt werden? Die Einordnung dieses Befundes erscheint insbesondere deshalb schwierig, weil Untersuchungen zum Zusammenhang zwischen der Computerspielnutzungsbiographie und späteren kognitiven Leistungen bislang noch nicht durchgeführt wurden. Daher sollen an dieser Stelle, auch aufgrund des explorativen Charakters der Datenbasis, nur sehr vorsichtige Interpretationen angeboten werden: Zum einen könnte der gegenläufige Befund in Hinblick auf die Konzentrationsleistung als weiterer Hinweis dafür verstanden werden, dass die Annahme einer unmittelbaren Beeinträchtigung der Konzentrationsleistung durch gewalthaltige Medien als zunehmend unwahrscheinlich gelten kann. So wäre hier gegenläufig zu den aufgefundenen Befunden zu erwarten gewesen, dass gerade bei Jungen Computerspielzeiten mit geringerer Konzentrationsleistung in Zusammenhang stehen, da diese weitaus häufiger als Mädchen gewalthaltige Spiele nutzen. Der Befund wiederum, dass nur bei Mädchen das Spielen in der Kindheit negativ mit kognitiven Leistungen korreliert, könnte als Hinweis darauf verstanden werden, dass ein intensives Spielverhalten von Mädchen im Kindesalter weit deutlicher als bei Jungen für eine risikobehaftete Entwicklung kennzeichnend ist. Somit könnte sich dieser Befund in der Weise als Artefakt entpuppen, dass die Kontrolle von kulturellem Kapital und Primärsozialisation nicht hinreichend ist, um bei Mädchen die mit erhöhten Spielzeiten verbundenen Risikomerkmale "herauszurechnen". Diese Interpretation ist möglicherweise konservativ, erscheint jedoch angesichts des explorativen Charakters der Auswertungsmethode und dem Mangel an vergleichbaren Daten an dieser Stelle am ehesten vertretbar.

Die Analysen zum Zusammenhang von aktuellen Mediennutzungsmustern und kognitiver Leistungsfähigkeit im Erwachsenenalter weisen noch einmal auf die Wichtigkeit der Unterscheidung von Nutzungsinhalten in Wirkungsstudien hin, die eine Beschreibung der kognitiven Wirkungen von Unterhaltungsmedien zum Ziel haben (vgl. Tabelle 37). Werden in den Partialkorrelationen unter Berücksichtigung von verbaler Intelligenz und Trait-Aggressivität nur die Medienzeiten betrachtet, ergeben sich kaum bedeutsame Zusammenhänge mit den untersuchten Leistungsparametern. Nur bei Frauen weist sowohl die allgemeine als auch die 
gewaltneutrale Spielzeit signifikant positive Korrelationen zur Lernleistung auf. Dieser Befund liefert eine weitere Bestätigung der bereits diskutierten Annahme (siehe oben), dass bei Frauen die gewaltneutrale Computerspielzeit durch die allgemeine Computerspielzeit verhältnismäßig akkurat abgebildet wird. Bei differenzierter Betrachtung der Inhalte zeigt sich hingegen, dass hinsichtlich der neutralen Medienzeit nur positive, hinsichtlich der gewalthaltigen Medienzeit nur negative Zusammenhänge mit den kognitiven Leistungen aufgefunden werden. So zeigt sich bei Männern der positive Zusammenhang von Computerspielnutzung und Konzentrationsleistung, der auch schon biographisch aufgezeigt werden konnte (siehe oben), nur für die Nutzung gewaltneutraler, nicht jedoch gewalthaltiger Inhalte. Zudem ergibt sich für Männer, dass nur die Nutzung gewalthaltiger nicht jedoch gewaltneutraler Spiele mit geringerer verbaler Gedächtnisleistung in $\mathrm{Zu}$ sammenhang steht. Dass den Inhalten der genutzten Medien auch im Erwachsenenalter noch diese differenzielle Bedeutung zukommt erscheint bemerkenswert und gilt bislang in der Forschung, zumindest in Hinblick auf die Computerspielnutzung, als höchst umstritten (vgl. Kapitel 1.3.1). So wurde beispielsweise postuliert, dass die Nutzung von Computerspielen die räumlich-visuelle Gedächtnisleistung verbessern kann (vgl. Boot, et al., 2008; Ferguson, et al., 2008). Hinsichtlich allgemeiner räumlich-visueller Leistungen wurde sogar postuliert, dass die Nutzung gewalthaltige Spiele aufgrund der elaborierteren Darstellung räumlicher Zusammenhänge ein besonders hohes Förderungspotential aufweist (vgl. Barlett, Vowels, et al., 2009). In der vorliegenden Untersuchung stehen jedoch weder neutrale noch gewalthaltige Computerspielzeit mit einer erhöhten räumlichen $\mathrm{Ge}-$ dächtnisleistung in Verbindung. Stattdessen finden sich bei Männern negative Zusammenhänge zwischen der Computerspielnutzung und der verbalen Gedächtnisleistung, auch unter Kontrolle von verbaler Intelligenz und Trait-Aggressivität. $\mathrm{Ob}$ in diesem Befundmuster eine zaghafte Bestätigung der von Greenfield (2009) formulierten Annahme eines "Trade-Offs" zwischen sprachbezogenen und visuellräumlichen Leistungsaspekten zum Ausdruck kommen könnte, welcher der zunehmenden Nutzung digitaler Medien geschuldet ist, kann an dieser Stelle auf Basis der Daten jedoch nicht abschließend geklärt werden.

\subsubsection{Methodenkritik}

Eine vieldiskutierte Achillesferse von Laborexperimenten ist die externe Validität der generierten Untersuchungsbefunde. Hierbei sind, bezogen auf die vorliegende Untersuchung, insbesondere die Grundprobleme anzusprechen, die mit dem Einsatz von Unterhaltungsmedien in Laborexperimenten verbunden sind: Probanden müssen sich vorgegebenen Angeboten aussetzen und können diese nicht entspre- 
chend ihrer Interessen und ihrer aktuellen Stimmungslage selbstbestimmt auswählen (vgl. Kapitel 2.1.2). Hier kann der vorliegenden Untersuchung sicherlich zugutegehalten werden, dass immerhin verschiedene Maßnahmen ergriffen wurden, um diesem Problem zu begegnen: So wurden pro Bedingung drei Angebote aus verschiedenen Genrekategorien bereitgestellt, um unterschiedliche Interessenlagen abdecken zu können. Zudem wurde versucht, das Rezeptionserleben möglichst natürlich zu gestalten, indem auf physiologische Messungen verzichtet wurde, ein geschützter und ungezwungener Rahmen bei der Medienrezeption vermittelt wurde und die Rolle des "Lehrers" (Versuchsleiter) von der des "Unterhaltungsanimateurs" (Assistenz) getrennt wurde. Zudem wurde bei der Wahl des Stimulusmaterials Wert darauf gelegt, dass hochgradig involvierende und hinsichtlich der Spiele mittelschwere Mainstreamangebote mit schneller Zugänglichkeit bereitgestellt werden. Durch vergleichsweise lange Rezeptionsphasen (3 x 20 Minuten) sollte den Versuchspersonen zudem ein intensives Eintauchen in die Medienwelten ermöglicht werden. Trotz dieser Maßnahmen kann jedoch auch für die vorliegende Untersuchung nicht beansprucht werden, dass alltägliche Unterhaltungsmediennutzung originalgetreu abgebildet wurde. Die Maßnahmen sind damit lediglich als Bemühungen zur Optimierung der externen Validität zu verstehen.

Eine ganz ähnliche Frage der externen Validität kann hinsichtlich der motivationalen Gegebenheiten schulischen Lernens aufgeworfen werden. So muss kritisch hinterfragt werden, ob in der Schule zu erbringende kognitive Leistungen in Laborexperimenten überhaupt mit ausreichender externer Validität "simuliert" werden können. Es gilt als allgemein beobachtetes Phänomen, dass Versuchspersonen in Lernexperimenten besonders hoch motiviert sind (vgl. Baddeley, 1997, S. 107). Übereinstimmend mit dieser Erkenntnis fällt auch die Selbsteinschätzung der Arbeitsmotivation in der vorliegenden Untersuchungen verhältnismäßig hoch aus (vgl. Kapitel 2.2.2, Tabelle 20). Eine geringere Arbeitsmotivation, wie sie zum Beispiel in der Schule in besonders unliebsamen Fächern durchaus gegeben sein kann, könnte zu einer geringeren Qualität der Encodierung und damit zu besonders instabilen Wissensrepräsentationen führen, denen in Hinblick auf die Konsolidierung eine besonders hohe Störanfälligkeit unterstellt werden kann. Derartige Überlegungen können jedoch im Rahmen von Laborexperimenten nur dann sinnvoll überprüft werden, wenn Arbeitsmotivation seinerseits systematisch manipuliert wird (z. B. durch eine Variation der motivationalen Anreizebene). In der vorliegenden Untersuchung wurde hierauf verzichtet, da der Einbezug eines weiteren Manipulationsfaktors die Komplexität des Designs noch weiter erhöht hätte und dieser zudem versuchsökonomische Probleme hinsichtlich der einzubeziehenden Stichprobengröße sowie der Gesamtdauer des Versuchs bedingt hätte. Stattdessen wurde versucht, durch die Gegenüberstellung einer vergleichsweise monotonen und langandauernden schulischen Lern- und Testsituation, die immer wieder durch 
vergleichsweise spannende Freizeitphasen unterbrochen wird, ein motivationales Gefälle von "Arbeit und Prüfung" auf der einen und "Freizeitvergnügen" auf der anderen Seite herzustellen, was in ähnlicher Weise auch von Schülern erlebt werden kann. Es kann jedoch selbstverständlich nicht zuverlässig eingeschätzt werden, ob damit schulische Lernprozesse hinreichend valide abgebildet werden konnten.

Eine methodisch operationale Einschränkung im gewählten Untersuchungsdesign kann darin gesehen werden, dass Distresserleben allein mittels subjektiver Selbsteinschätzungsdaten erfasst wurde. Physiologische Daten hätten möglicherweise, zumindest unter der Voraussetzung breitbandiger Messansätze, differenziertere Auswertungen zu den Prädiktoren von Distresserleben ermöglicht und hierbei auch methodenvergleichende Analysen zwischen subjektiven und objektiven Maßen ermöglicht. Mit solchen Daten hätte somit die Aussagekraft der Untersuchung zum emotionalen Wirkpotential gewalthaltiger Medien erhöht werden können. Auch hätte beispielsweise eine Bestimmung des Cortisolspiegels über die Entnahme von Speichelproben dafür herangezogen werden können, post-hoc physiologische "High-Responder" zu identifizieren, die in besonderer Weise mit einer Reaktion der HPA-Achse (vgl. Kapitel 1.4.2) auf die Mediengewalt reagiert haben. Für die Überprüfung der kognitiven Wirkhypothesen dieser Untersuchung ist dies aber zumindest nicht von zentraler Bedeutung, da hierfür ohnehin keine Post-HocMaße genutzt werden sollten. Eine Post-Hoc-Identifikation sogenannter "HighResponder" und eine nachträgliche Analyse möglicher kognitiver Leistungsbeeinträchtigungen in dieser Subgruppe - wie sie in vielen aufgefundenen Untersuchungen zur Stressforschung vorgenommen wurde (vgl. Kapitel 1.4.3) - ist zumindest dann als methodisch fragwürdig einzustufen, wenn der explorative Charakter derartiger Analysen nicht hinreichend kenntlich gemacht wird und derartige Unterschiede im Nachhinein plausibilisiert werden. Zudem hätte die Erfassung biopsychologischer Erlebniskorrelate auch eine Reihe von Nachteilen für die Versuchsökonomie gehabt und das Versuchspersonenverhalten in nicht intendierter Weise verfälschen können, weshalb den Nachteilen der nicht berücksichtigten physiologischen Messungen sicherlich auch eine Reihe von Vorteilen gegenüberstehen.

Ein ganz grundsätzliches und auch schon angesprochenes Problem der vorliegenden Untersuchung (vgl. Kapitel 2.1.1) stellt das Alter der einbezogenen Versuchspersonen dar. Wie bereits ausgeführt, kann aus den vorliegenden Daten nicht ohne Weiteres abgeleitet werden, dass eine Nutzung gewalthaltiger Medien im Kindes- oder Jugendalter ebenfalls keine beeinträchtigende Wirkung auf Gedächtnisfunktionen aufweist, da angenommen werden muss, dass erwachsene Person bei der Rezeption medialer Gewalt in weit geringerem Maße Distress erleben als kindliche oder jugendliche Rezipienten. Zwar kann angeführt werden, dass in der vorliegenden Untersuchung auch bei erwachsenen Personen, die Gewaltmedien ten- 
denziell ablehnen, keine Beeinträchtigungen aufgefunden wurden und dass auch die explorativen Post-Hoc-Analysen keine Hinweise dafür liefern, dass die kognitiven Leistungen bei Personen mit hohem Distresserleben geringer ausfallen (vgl. Anhang K). Gerade vor dem Hintergrund, dass insbesondere im Kindesalter gravierende Angstreaktionen in Konfrontation mit gewalthaltigen Medien berichtet werden (vgl. Kapitel 1.3.3.2.2), muss jedoch offen bleiben, ob die Aussagen dieser Untersuchung auf minderjährige Rezipienten übertragen werden können.

Ein letzter Einwand kann in Hinblick auf kurzfristige und langfristige Wirkannahmen formuliert werden. Laborexperimente haben zwar den großen Vorteil, kausale Wirkannahmen überprüfen und Störeinflüsse effektiv kontrollieren zu können. Langfristige Wirkprozesse können in Experimenten jedoch nicht abgebildet werden. Die zusätzlich geleisteten quasiexperimentellen Analysen liefern weitergehende Hinweise zu der Frage, wie Bildschirmmediennutzung und Kognition langfristig miteinander in Beziehung stehen könnten. Diese Daten können jedoch selbst dann nicht als kausale Wirkungen interpretiert werden, wenn dabei wie im vorliegenden Fall im Rahmen von Partialkorrelationen andere wesentliche Einflussgrößen kontrolliert werden. Sie ersetzen somit keine längsschnittlichen Daten zur Wirkung von Mediennutzungsmenus auf spätere kognitive Leistungsparameter. Eine weitere Einschränkung muss darin gesehen werden, dass in der vorliegenden Untersuchung hinsichtlich biographischer Mediennutzungsparameter nicht die Inhalte der Mediennutzung erfasst wurden (vgl. Kapitel 2.2.5.1; Fußnote 93). Leider stehen hierzu keine etablierten Instrumente zur Verfügung und es bestehen nachwievor erhebliche Zweifel daran, ob sich derartige Informationen retrospektiv überhaupt verlässlich ermitteln lassen. Dies erschwert jedoch die Interpretation der Zusammenhangsdaten insofern, dass sich selbst bei aktuellen Mediennutzungsparametern im Erwachsenenalter noch relevant Unterschiede dahingehend ergeben, ob gewalthaltige oder gewaltneutrale Inhalte genutzt werden. Insgesamt erscheint die Annahme berechtigt, dass inhaltliche Unterschiede im Mediennutzungsmenu von Kindern und Jugendlichen noch gravierenderen Einfluss darauf haben sollten, ob sie sich kognitiv stimulierend oder beeinträchtigend auswirken.

\subsubsection{Ausblick und Implikationen für die Forschung}

Gerade aufgrund der geringen Zahl von Studien zum differenziellen affektiven und kognitiven Wirkpotential von gewalthaltigen Filmen und Computerspielen erscheinen weitere Untersuchungen notwendig, um zur Theorieentwicklung in diesem Feld beizutragen. In diesem Zusammenhang sollte auch bedacht werden, dass die Technik seit Durchführung der vorliegenden Untersuchung im Jahr 2006 bedeutende Fortschritte gemacht hat, indem technologische Weiterentwicklungen der 
Computerhardware und Next-Generation-Spielkonsolen wie die Playstation 3 oder die XBOX 360 auf dem Markt erschienen sind. Damit kommt der Realitätsgrad der grafischen Darstellungen von Computerspielen nunmehr, im Jahr 2010, filmischen Darstellungen bereits weitaus näher als noch im Jahr der Studiendurchführung. Vor dem Hintergrund der rasanten technischen Entwicklung, die nicht nur auf visuelle und akustische Darstellungsmittel beschränkt ist sondern auch eine Zunahme der Komplexität von Spielkonzeptionen umfasst, können systematische Untersuchung des emotionalen und kognitiven Wirkpotentials interaktiver Unterhaltungsmedien auch in Zukunft als dringend erforderlich bewertet werden.

Neben experimenteller medienpsychologischer Wirkungsforschung ist hierbei ein dringender Bedarf an längsschnittlichen Untersuchungen anzumelden. Bislang existieren im Gegensatz zum Fernsehen noch keine umfassenderen Längsschnittdaten zu den Wirkungen von Computerspielnutzung auf kognitive und schulische Leistungsparameter (für eine zwischenzeitlich erschienene Längsschnittstudie vgl. Mößle et al., 2010). Die geringen Forschungsanstrengungen sind möglicherweise dadurch zu erklären, dass Computerspielnutzung kein typisches frühkindliches Phänomen darstellt und das Wirkpotential dieses Formates deshalb gegenüber dem Fernsehen weitaus weniger im Fokus entwicklungspsychologischer Überlegungen stand. Dies scheint jedoch angesichts der zunehmenden Verbreitung interaktiver Unterhaltungsmedien sowie in Hinblick auf die Tatsache, dass kognitive Wirkungen von Computerspielen noch im Erwachsenenalter nachgewiesen wurden, kein überzeugender Standpunkt mehr zu sein. Stattdessen kann eine Verknüpfung von Schulleistungsforschung und der Untersuchung kognitiver Entwicklungsverläufe vor dem Hintergrund unterschiedlicher Medienmenus als besonders vielversprechend bewertet werden. Nachwievor ist nicht geklärt, wie der negative Zusammenhang von Bildschirmmediennutzung und Schulleistung erklärt werden kann. Zudem erscheint die Forschung zum Wirkpotential gewalthaltiger Medien auf aggressionsbezogene Konstrukte bislang relativ losgelöst von Wirkvermutungen, die kognitiv-leistungsbezogene und schulische Auswirkungen betreffen (vgl. Kapitel 1.5.1). Zukünftige Forschung sollte eine Verbindung von Theorien zum emotionalen und kognitiven Wirkpotential von Bildschirmmediennutzung anstreben und hier bislang voneinander losgelöste Ansätze und Paradigmen integrieren.

Da in der vorliegenden Untersuchung erwachsene Versuchspersonen untersucht wurden und die einzige aufgefundene publizierte Untersuchung zum Kindesalter gegenläufige Befunde erbracht hat (vgl. Dworak, et al., 2007) kann die grundsätzliche Empfehlung ausgesprochen werden, eine Untersuchung ähnlich der vorliegenden Art auch an Kindern durchzuführen, um gerade für diese im Zentrum des Interesses stehende Gruppe eine aussagekräftigere Datenbasis zur Verfügung zu stellen. Hierzu müssten jedoch unbedingt engagierte forschungsethische Überlegungen in Hinblick auf das einzusetzende Stimulusmaterial angestellt werden. 
Dieses angesprochene Dilemma wird nicht ohne Weiteres zu lösen sein, da es forschungsethisch auf keinen Fall vertretbar wäre, Kinder mit Medien zu konfrontieren, die der in dieser Untersuchung angewendeten Mediengewaltdefinition entsprechen. Neben laborexperimentellen Ansätzen könnten auch feldexperimentelle Untersuchungen in Erwägung gezogen werden, die an dem angesprochenen Problem der geringeren externen Validität von Laborexperimenten anzuknüpfen. Diese hätten den Vorteil, dass lebenswirkliche schulische Lernprozesse zur Grundlage genommen werden könnten und das freizeitliche Mediennutzungsverhalten gleichzeitig systematisch manipuliert würde, welches dabei jedoch weiterhin im natürlichen Sozialisationskontext des Schülers verbleibt. Aber auch derartige Überlegungen erfordern unbedingt eingehende forschungsethische Debatten darüber, welche Eingriffe hier zum Zwecke wissenschaftlicher Forschung noch vertretbar erscheinen. 


\section{Literaturverzeichnis}

Amaral, D. G. (2000). The anatomical organization of the central nervous system. In E. R. Kandel, J. H. Schwartz \& T. M. Jessell (Eds.), Principles of neural science (Vol. 4): The McGraw-Hill Companies.

Amthauer, R., Brocke, B., Liepmann, D., \& Beauducel, A. (2001). I-S-T 2000 R: IntelligenzStruktur-Test 2000 R. Göttingen, Bern, Toronto, Seattle: Hogrefe.

Anand, Vivek (2007). A Study of Time Management: The Correlation between Video Game Usage and Academic Performance Markers. CyberPsychology \& Behavior, 10(4), 552-559.

Anderson, C. A., \& Bushman, B. J. (2001). Effects of violent video games on aggressive behavior, aggressive cognition, aggressive affect, physiological arousal, and prosocial behavior: A meta-analytic review of the scientific literature. Psychological Science, $12(5), 353$.

Anderson, C. A., Carnagey, N. L., Flanagan, Mindy, Benjamin, A.J., Eubanks, Janie, \& Valentine, Jeffrey C. (2004). Violent Video Games: Specific Effects of Violent Content on Aggressive Thoughts and Behavior. Advances in Experimental Social Psychology, 36, 200-246.

Anderson, Craig A. (2004). An update on the effects of playing violent video games. Journal of Adolescence, 27(1), 113-122.

Anderson, Craig A., \& Dill, Karen E. (2000). Video Games and Aggressive Thoughts, Feelings, and Behavior in the Laboratory and in Life. Journal of Personality and Social Psychology, 78(4), 772-790.

Anderson, D. R., Huston, A.C., Schmitt, K.L., Linebarger, D.L., \& Wright, J.C. (2001). Early childhood television viewing and adolescent behavior. Monographs of the Society for Research in Child Development, 66(1), 1-143.

Anderson, J. R. (1996). Kognitive Psychologie (Vol. 2). Heidelberg, Berlin, Oxford: Spektrum Akademischer Verlag.

Andresen, B. (2002). Hamburger Persönlichkeitsinventar: HPI. Göttingen: Hogrefe Verlag für Psychologie.

Andrews, Glenda, \& Murphy, Karen (2006). Does Video Game Playing Improve Executive Function? In Michael A. Vanchevsky (Ed.), Frontiers of Cognitive Psychology (pp. 145-161).

Ashcraft, M. H. (2002). Math anxiety: personal, educational, and cognitive consequences. Current Directions in Psychological Science, 11(5), 181-185.

Baddeley, A. (1990). Human memory: Theory and practice. Boston: Allyn \& Bacon.

Baddeley, A. (1997). Human Memory: Theory and Practice: Psychology Press Ltd.

Baddeley, A. (2000). The episodic buffer: A new component of working memory. Trends in Cognitive Science, 4(11), 417-423.

Baddeley, A., \& Hitch, G. (1974). Working memory. In G. A. Bower (Ed.), The psychology of learning and motivation (pp. 48-79): Academic Press. 
Baier, D., \& Rehbein, F. (2009). Computerspielabhängigkeit im Jugendalter. In C. J. Tuly (Ed.), Multilokalität und Vernetzung: Beiträge zur technikbasierten Gestaltung jugendlicher Sozialräume (pp. 139-155). Weinheim, München: Juventa Verlag.

Baldaro, B., Tuozzi, G., Codispoti, M., Montebarocci, O., Barbagli, F., Trombini, E., et al. (2004). Aggressive and non-violent videogames: Short-term psychological and cardiovascular effects on habitual players. Stress \& Health: Journal of the International Society for the Investigation of Stress, 20(4), 203-208.

Ballard, M. E., \& Wiest, J. R. (1996). Mortal Kombat (tm): The effects of violent videogame play on males' hostility and cardiovascular responding. Journal of Applied Social Psychology, 26(8), 717-730.

Barlett, C. P., Branch, O., Rodeheffer, C. D., \& Harris, R. (2009). How long do the shortterm violent video game effects last? Aggress Behav, 35(3), 225-236.

Barlett, C. P., \& Rodeheffer, C. D. (2009). Effects of realism on extended violent and nonviolent video game play on aggressive thoughts, feelings, and physiological arousal. Aggressive Behavior, 35(3), 213-224.

Barlett, C. P., Vowels, C. L., Shanteau, J., Crow, J., \& Miller, T. (2009). The effects of violent and non-violent computer games on cognitive performance. Computers in Human Behavior, 25, 96-102.

Bartholow, Bruce D., Bushman, Brad J., \& Sestir, Marc A. (2006). Chronic violent video game exposure and desensitization to violence: Behavioral and event-related brain potential data. Journal of Experimental Social Psychology, 42(4), 532-539

Baumert, J., Artelt, C., Carstensen, C. H., Sibberns, H., \& Stanat, P (2001). Untersuchungsgegenstand, Fragestellungen und technische Grundlagen der Studie. In J. Baumert, E. Klieme, M. Neubrand, M. Prenzel, U. Schiefele, W. Schneider, P. Stanat, K.-J. Tillmann \& M. Weiß (Eds.), PISA 2000. Basiskompetenzen von Schülerinnen und Schülern im internationalen Vergleich. Opladen: Leske und Budrich.

Baumert, J., Artelt, C., Klieme, E., Neubrand, M., Prenzel, M., Schiefele, U., et al. (Eds.). (2002). PISA 2000 - Die Länder der Bundesrepublik Deutschland im Vergleich. Opladen: Leske + Budrich.

Bednorz, P, \& Schuster, M. (2002). Einführung in die Lernpsychologie (Vol. 3). München: Ernst Reinhardt.

Beentjes, J. W. J., \& Van der Voort, T. H. A. (1988). Television's impact on children's reading skills: A review of research. Reading Research Quarterly, 23, 389-413.

Beilock, S. L., \& Decaro, M. S. (2007). From poor performance to success under stress: working memory, strategy selection, and mathematical problem solving under pressure. J Exp Psychol Learn Mem Cogn, 33(6), 983-998.

Birbaumer, N., \& Schmidt, R. F. (1999). Biologische Psychologie (Vol. 4). Berlin, Heidelberg, New York: Springer.

Bonfadelli, H. (2004). Medienwirkungsforschung I (Vol. 3). Konstanz: UVK Verlagsgesellschaft.

Boot, W. R., Kramer, A. F., Simons, D. J., Fabiani, M., \& Gratton, G. (2008). The effects of video game playing on attention, memory, and executive control. Acta Psychol (Amst), 129(3), 387-398.

Bortz, J, \& Döring, N. (2003). Forschungsmethoden und Evaluation (Vol. 3). Berlin, Heidelberg, New York: Springer. 
Borusiak, P., Bouikidis, A., Liersch, R., \& Russell, J. B. (2008). Cardiovascular effects in adolescents while they are playing video games: A potential health risk factor? Psychophysiology, 45, 327-332.

Bos, W., Lankes, E.-M., Prenzel, M., Schwippert, K., Walther, G., \& Valtin, R. (Eds.). (2003). Erste Ergebnisse aus IGLU. Schülerleistungen am Ende der vierten Jahrgangsstufe im internationalen Vergleich. Münster: Waxman.

Bourdieu, P. (1983). Ökonomisches Kapital, kulturelles Kapital, soziales Kapital. In R. Kreckel (Ed.), Soziale Welt. Sonderband 2: Soziale Ungleichheiten (pp. 183-198). Göttingen: Otto Schwartz \& Co.

Bourne, L. E., \& Yaroush, R. A. (2003). Stress and cognition: a cognitive psychological perspective. Boulder, Colorado: University of Colorado: Department of Psychology.

Brady, S. S., \& Matthews, K. A. (2006). Effects of media violence on health-related outcomes among young men. Arch Pediatr Adolesc Med, 160(4), 341-347.

Breitenstein, C., \& Knecht, S. (2002). Development and validation of a language learning model for behavioral and functional-imaging studies. Journal of Neuroscience Methods, 114(2), 173-180.

Buckley, K. E., \& Anderson, C. A. (2006). A theoretical model of the effects and consequences of playing video games. In P. Vorderer \& J. Bryant (Eds.), Playing video games: motives, responses, and consequences (pp. 363-378). Mahwah: NJ:Erlbaum.

Bühner, M. (2006). Einführung in die Test- und Fragebogenkonstruktion (Vol. 2.). München: Pearson Studium.

Buehner, M., Mangels, M., Krumm, S., \& Ziegler, M. (2005). Are working memory and attention related constructs? Journal of Individual Differences, 26(3), 107-120.

Bundesverband Interaktive Unterhaltungssoftware e.V. (2006). Unterhaltungssoftwareindustrie 2005/2006. Retrieved 6.1.2010: http://www.biu-online.de/fileadmin/user/dateien/Marktzahlen_2005.pdf

Bundesverband Interaktive Unterhaltungssoftware e.V. (2009). Marktzahlen Computer- und Videospiele Gesamtjahr 2008. Retrieved 6.1.2010: http:/www.biu-online.de/fileadmin/ user/dateien/BIU_Marktzahlen_Gesamtjahr_2008.pdf

Bushman, B. J., \& Anderson, C. A. (2002). Violent video games and hostile expectations: A test of the general aggression model. Personality \& Social Psychology Bulletin, 28(12), 1679-1686.

Bushman, B. J., \& Bonacci, A. M. (2002). Violence and sex impair memory for television ads. Journal of Applied Psychology, 87(3), 557-564.

Bushman, B. J., \& Geen, R. G. (1990). Role of Cognitive-Emotional Mediators and Individual Differences in the Effects of Media Violence on Aggression. Journal of Personality and Social Psychology, 58(1), 156-163.

Bushman, B. J., \& Huesmann, L. R. (2006). Short-term and long-term effects of violent media on aggression in children and adults. Archives of Pediatrics \& Adolescent Medicine, 160(4), 348-352.

Cahill, L., \& McGaugh, J. L. (1996). Modulation of memory storage. Current Opinion in Neurobiology, 2, 237-242.

Cantor, J. (2001). The media and children's fears, anxieties, and perception of danger. In D. G. Singer \& J. L. Singer (Eds.), Handbook of children and the media. Thousand Oaks, London, New Delhi: Sage Publications. 
Carnagey, N. L., \& Anderson, C. A. (2005). The effects of reward and punishment in violent video games on aggressive affect, cognition, and behavior. Psychological Science, 16(11), 882-889.

Carnagey, N. L., Anderson, C. A., \& Bushman, B. J. (2007). The effect of video game violence on psychological desensitization to real-life violence. Journal of Experimental Social Psychology, 43(3), 489-496.

Castel, A. D., Pratt, J., \& Drummond, E. (2005). The effects of action video game experience on the time course of inhibition of return and the efficiency of visual search. Acta Psychologica, 119(2), 217-230.

Chan, P. A., \& Rabinowitz, T. (2006). A cross-sectional analysis of video games and attention deficit hyperactivity disorder symptoms in adolescents. Annals of General Psychiatry, 5, 16.

Christakis, D. A., Zimmerman, F. J., DiGiuseppe, D. L., \& McCarty, C. A. (2004). Early television exposure and subsequent attentional problems in children. Pediatrics, 113(4), 708-713.

Cohen, J. (1988). Statistical power analysis for the behavioral sciences (Vol. 2). Hillsdale, New York: Erlbaum.

Comstock, G., \& Scharrer, E. (1999). Scholastic Performance. In G. Comstock \& E. Scharrer (Eds.), Television: What's On, Who's Watching, and What It Means (pp. 227-264). San Diego, CA: Academic Press.

Cowan, N. (1999). An Embedded-Processes Model of Working Memory Models of working memory : mechanisms of active maintenance and executive control (pp. 62-101). Cambridge [u.a.]: Cambridge Univ. Press.

Crönlein, Tatjana, Stanggassinger, Daniela, Geisler, Peter, Popp, Roland, Zulley, Jürgen, \& Lukesch, Helmut (2007). Fernesehkonsum und Schlafstörungen bei Kindern. Psychiatrische Praxis, 34, 59-61.

Cummings, Hope M., \& Vandewater, Elizabeth A. (2007). Relation of Adolescent Video Game Play to Time Spent in Other Activities. Archives of Pediatrics \& Adolescent Medicine, 161(7), 684-689.

Datenreport 2004: Zahlen und Fakten über die Bundesrepublik Deutschland (2005). Bonn: Bundeszentrale für politische Bildung.

De Lisi, R., \& Cammarano, D. M. (1996). Computer experience and gender differences in undergratuate mental rotation performance. Computers in Human Behavior, 12(3), 351-361.

De Lisi, R., \& Wolford, J. L. (2002). Improving children`s mental rotation accuracy with computer game playing. Journal of Genetic Psychology, 163(3), 272-282.

De Quervain, D. J. F., Aerni, A., Schelling, G., \& Roozendaal, B. (2009). Glucocorticoids and the regulation of memory in health and disease. Frontiers in Neuroendocrinology, 30, 358-370.

De Quervain, D. J. F., Henke, K., Aerni, A., Treyer, V., McGaugh, J. L., Berthold, T., et al. (2003). Glucocorticoid-induced impairment of declarative memory retrieval is associated with reduced blood flow in the medial temporal lobe. European Journal of Neuroscience, 17(6), 1296-1302.

De Quervain, D. J. F., Roozendaal, B., Nitsch, R. M., McGaugh, J. L., \& Hock, C. (2000). Acute cortisone administration impairs retrieval of long-term declarative memory in humans. Nat Neurosci, 3, 313-314. 
Dedovic, K., Duchesne, A., Andrews, J., Engert, V., \& Pruessner, J. C. (2009). The brain and the stress axis: The neural correlates of cortisol regulation in response to stress. NeuroImage, 47, 864-871.

Diamond, D. M., Campbell, A. M., Park, C. R., Halonen, J., \& Zoladz, P. R. (2007). The temporal dynamics model of emotional memory processing: a synthesis on the neurobiological basis of stress-induced amnesia, flashbulb and traumatic memories, and the Yerkes-Dodson law. Neural Plast, 2007, 1-33.

Dickerson, S. S., \& Kemeny, M. E. (2004). Acute stressors and cortisol responses: a theoretical integration and synthesis of laboratory research. Psychol Bull, 130(3), 355-391.

Dietz, Tracy L. (1998). An Examination of Violence and Gender Role Portrayals in Video Games: Implications for Gender Socialization and Aggressive Behavior. Sex Roles, $38(5 / 6), 425-442$.

Dworak, M., Schierl, T., Bruns, T., \& Struder, H. K. (2007). Impact of singular excessive computer game and television exposure on sleep patterns and memory performance of school-aged children. Pediatrics, 120(5), 978-985.

Dye, M. W. G., Green, C. S., \& Bavelier, D. (2009). The development of attention skills in action video game players. Neuropsychologia, 47(8-9), 1780-1789.

Ennemoser, Marco (2007). Sprachentwicklung und Schriftspracherwerb in der Mediengesellschaft: Wirkungspotenziale von Printmedien über Hörkassetten bis hin zur Fernsehund Computernutzung. L.O.G.O.S.- Interdisziplinär, 15(3), 191-199

Ennemoser, Marco, \& Schneider, Wolfgang (2007). Relations of Television Viewing and Reading. Journal of Educational Psychology, 99(2), 349-368.

Erdfelder, E., Buchner, A., Faul, F., \& Brandt, M. (2004). GPOWER: Teststärkeanalysen leicht gemacht. In E. Erdfelder \& J. Funke (Eds.), Allgemeine Psychologie und deduktivistische Methodologie. Göttingen: Vandenhoeck \& Ruprecht.

Erdfelder, E., Faul, F., \& Buchner, A. (1996). GPOWER: A general power analysis program. Behavior Research Methods, Instruments, and Computers, 28, 1-11.

Faul, F., Erdfelder, E., Lang, A.-G., \& Buchner, A. (2007). G*Power 3: A flexible statistical power analysis for the social, behavioral, and biomedical sciences. Behavior Research Methods, 39(2), 175-191.

Feierabend, S., \& Klingler, W. (1998). JIM 98 - Jugend, Information, (Multi-)Media. Basisuntersuchung zum Medienumgang 12 bis 19jähriger in Deutschland. Baden-Baden: Medienpädagogischer Forschungsverbund Südwest.

Feierabend, S., \& Klingler, W. (1999). JIM 99/2000 - Jugend, Information, (Multi-)Media. Basisuntersuchung zum Medienumgang 12 bis 19jähriger in Deutschland. Baden-Baden: Medienpädagogischer Forschungsverbund Südwest.

Feierabend, S., \& Klingler, W. (2000). JIM 2000 - Jugend, Information, (Multi-)Media. Basisuntersuchung zum Medienumgang 12 bis 19-Jähriger in Deutschland. Baden-Baden: Medienpädagogischer Forschungsverbund Südwest.

Feierabend, S., \& Klingler, W. (2001). JIM-Studie 2001 - Jugend, Information, (Multi-)Media. Basisuntersuchung zum Medienumgang 12 bis 19-Jähriger in Deutschland. BadenBaden: Medienpädagogischer Forschungsverbund Südwest.

Feierabend, S., \& Klingler, W. (2002). JIM-Studie 2002 - Jugend, Information, (Multi-)Media. Basisuntersuchung zum Medienumgang 12 bis 19-Jähriger in Deutschland. BadenBaden: Medienpädagogischer Forschungsverbund Südwest. 
Feierabend, S., \& Klingler, W. (2003). JIM-Studie 2003 - Jugend, Information, (Multi-)Media. Basisuntersuchung zum Medienumgang 12 bis 19-Jähriger in Deutschland. BadenBaden: Medienpädagogischer Forschungsverbund Südwest.

Feierabend, S., \& Klingler, W. (2008). Was Kinder sehen. Eine Analyse der Fernsehnutzung Drei- bis 13-Jähriger. Media Perspektiven, 4, 190-204.

Feierabend, S., \& Mohr, I. (2004). Mediennutzung von Klein- und Vorschulkindern. Ergebnisse der ARD/ZDF-Studie "Kinder und Medien 2003". [PDF]. Media Perspektiven, 9, 453-461.

Feierabend, S., \& Rathgeb, T. (2004). JIM-Studie 2004 - Jugend, Information, (Multi-)Media. Basisuntersuchung zum Medienumgang 12 bis 19-Jähriger in Deutschland. BadenBaden: Medienpädagogischer Forschungsverbund Südwest.

Feierabend, S., \& Rathgeb, T. (2005). JIM-Studie 2005 - Jugend, Information, (Multi-)Media. Basisuntersuchung zum Medienumgang 12 bis 19-Jähriger in Deutschland. Stuttgart: Medienpädagogischer Forschungsverbund Südwest.

Feierabend, S., \& Rathgeb, T. (2006). JIM-Studie 2006 - Jugend, Information, (Multi-)Media. Basisuntersuchung zum Medienumgang 12 bis 19-Jähriger in Deutschland. Stuttgart: Medienpädagogischer Forschungsverbund Südwest.

Feierabend, S., \& Rathgeb, T. (2007). JIM-Studie 2007 - Jugend, Information, (Multi-)Media. Basisuntersuchung zum Medienumgang 12 bis 19-Jähriger in Deutschland. Stuttgart: Medienpädagogischer Forschungsverbund Südwest.

Feierabend, S., \& Rathgeb, T. (2008). JIM-Studie 2008 - Jugend, Information, (Multi-)Media. Basisuntersuchung zum Medienumgang 12 bis 19-Jähriger in Deutschland. Stuttgart: Medienpädagogischer Forschungsverbund Südwest.

Feierabend, S., \& Rathgeb, T. (2009 a). JIM-Studie 2009 - Jugend, Information, (Multi-)Media. Basisuntersuchung zum Medienumgang 12 bis 19-Jähriger in Deutschland. Stuttgart: Medienpädagogischer Forschungsverbund Südwest.

Feierabend, S., \& Rathgeb, T. (2009 b). KIM-Studie 2008 - Kinder + Medien, Computer + Internet. Basisuntersuchung zum Medienumgang 6- bis 13-Jähriger. Stuttgart: Medienpädagogischer Forschungsverbund Südwest.

Feng, J., Spence, I., \& Pratt, J. (2007). Playing an action video game reduces gender differences in spatial cognition. Psychological Science, 18(10), 850-855.

Ferguson, C. J., Cruz, A. M., \& Rueda, S. M. (2008). Gender, Video Game Playing Habits and Visual Memory Tasks. Sex Roles, 58(3-4), 279-286.

Fleming, M. J., \& Rickwood, D. J. (2001). Effects of violent versus nonviolent video games on children's arousal, aggressive mood, and positive mood. Journal of Applied Social Psychology, 31(10), 2047 - 2071.

Frindte, W., \& Obwexer, I. (2003). Ego-Shooter: Gewalthaltige Computerspiele und aggressive Neigungen. Zeitschrift für Medienpsychologie, 15, 140-148.

Fröhlich, W. D. (2000). Wörterbuch Psychologie (Vol. 23). München: Deutscher Taschenbuch Verlag.

Fuchs, T., \& Wößmann, L. (2004). Computers and student learning: Bivariate and multivariate evidence on the availability and use of computers at home and at school (Working Paper No. 1321).

Funk, J. B., Baldacci, H. B., Pasold, T, \& Baumgardner, J. (2004). Violence exposure in real-life, video games, television, movies, and the internet: is there desensitization? Journal of Adolescence, 27(1), 23-40. 
Gaab, J., Rohleder, N., Nater, U. M., \& Ehlert, U. (2005). Psychological determinants of the cortisol stress response: the role of anticipatory cognitive appraisal. Psychoneuroendocrinology, 30, 599-610.

Gaddy, G. D. (1986). Television's impact on high school achievement. Public Opinion Quarterly, 50, 340-359.

Gentile, D. A., Lynch, P. J., Linder, J. R., \& Walsh, D. A. (2004). The effects of violent video game habits on adolescent hostility, aggressive behaviors, and school performance. Journal of Adolescence, 27, 5-22.

Gentile, D. A., \& Walsh, D. A. (2002). A normative study of family media habits. Journal of Applied Developmental Psychology, 23, 157-178.

Glaubke, C.R., Miller, P., Parker, M.A., \& Espejo, E. (2001). Fair Play? Violence, Gender and Race in Video Games. Oakland, CA.

Gortmaker, S. L., Salter, C. A., Walker, D. K., \& Dietz, W. H. (1990). The impact of television viewing on mental aptitude and achievement: A longitudinal study. Public Opinion Quarterly, 54, 594-604.

Green, C. S., \& Bavelier, D. (2003). Action video game modifies visual selective attention. Nature, 423, 534-537.

Green, C. S., \& Bavelier, D. (2006 a). Effect of Action Video Games on the Spatial Distribution of Visuospatial Attention. Journal of Experimental Psychology: Human Perception and Performance, 32(6), 1465-1478.

Green, C. S., \& Bavelier, D. (2006 b). Enumeration versus multiple object tracking: the case of action video game players. Cognition, 101(1), 217-245.

Green, C. S., \& Bavelier, D. (2007). Action-Video-Game Experience Alters the Spatial Resolution of Vision. Psychological Science, 18(1), 88-94.

Greenfield, P. M. (2009). Technology and Informal Education: What Is Taught, What Is Learned. Science, 323(5910), 69-71.

Greenfield, P. M., Dewinstanley, P., Kilpatrick, H., \& Kaye, D. (1994). Action video games and informal education: effects on strategies for dividing visual attention. Journal of applied developmental psychology, 15, 105-123.

Grimm, J. (1996). Between anxiety and aggression, physiological and psychosocial effects of television violence. European Psychiatry, 11(4), 215.

Grimm, P., Kirste, K., \& Weiß, J. (2005). Gewalt zwischen Fakten und Fiktion. Eine Untersuchung von Gewaltdarstellungen im Fernsehen unter besonderer Berücksichtigung ihres Realitäts- bzw. Fiktionalitätsgrades. Berlin: Vistas.

Groebel, J., \& Gleich, U. (1993). Gewaltprofil des deutschen Fernsehprogramms. Eine Analyse des Angebots privater und öffentlich-rechtlicher Sender (Vol. 6). Opladen: Leske + Budrich.

Haertel, G. D., Walberg, H. J., \& Weinstein, T. (1983). Psychological models of educational performance: A theoretical synthesis of constructs. Review of Educational Research, 53(1), 75-91.

Hancox, R. J., Milne, B. J., \& Poulton, R. (2005). Association of television viewing during childhood with poor educational achievement. Archives of Pediatrics \& Adolescent Medicine, 159, 614-618.

Harris, M. B., \& Williams, R. (1985). Video games and school performance. Education, 105(3), 306-309. 
Harrison, K., \& Cantor, J. (1999). Tales from the screen: Unduring fright reactions to scary media. Medial Psychology, 1(2), 97-116.

Hébert, S., Béland, R., Dionne-Fournelle, O., Crête, M., \& Lupien, S. J. (2005). Physiological stress response to video-game playing: the contribution of built-in music. Life Sciences, 76, 2371-2380.

Heins, E., Seitz, C., Schuz, J., Toschke, A. M., Harth, K., Letzel, S., et al. (2007). Schlafenszeiten, Fernseh- und Computergewohnheiten von Grundschulkindern in Deutschland. Gesundheitswesen, 69(3), 151-157.

Heller, K. A. (1997). Individuelle Bedingungsfaktoren der Schulleistung: Literaturüberblick. In F. E. Weinert \& A. Helmke (Eds.), Entwicklung im Grundschulalter (pp. 183-201). Weinheim: Beltz.

Heller, K. A., \& Perleth, C. (2000). Kognitiver Fähigkeitstest für 4. bis 12. Klassen, Revision KFT 4-12+R; Manual. Göttingen: Beltz Test.

Helmke, \& Weinert (1997). Bedingungsfaktoren schulischer Leistungen. In Weinert (Ed.), Psychologie des Unterrichts. Göttingen: Hogrefe.

Helmke, A. (1997). Individuelle Bedingungsfaktoren der Schulleistung: Ergebnisse aus dem SCHOLASTIK-Projekt. In F. E. Weinert \& A. Helmke (Eds.), Entwicklung im Grundschulalter (pp. 203-216). Weinheim: Beltz.

Higuchi, S., Motohashi, Y., Liu, Y., \& Maeda, A. (2005). Effects of playing a computer game using a bright display on presleep physiological variables, sleep latency, slow wave sleep and REM sleep. Journal of Sleep Research, 14(3), 267-273.

Hoppe-Graff, S., \& Kim, H.-O. (2002). Die Bedeutung der Medien für die Entwicklung von Kindern und Jugendlichen. In R. Oerter \& L. Montada (Eds.), Entwicklungspsychologie (Vol. 5, pp. 907-922). Weinheim: Belz.

Höynck, T., Mößle, T, Kleimann, M., \& Rehbein, F. (2007). Alterseinstufung von Computerspielen durch die USK in der Kritik. Politik und Kultur. Zeitung des Deutschen Kulturrats, 04, 11-12.

Höynck, T., Mößle, T., Kleimann, M., Pfeiffer, C., \& Rehbein, F. (2007). Jugendmedienschutz bei gewalthaltigen Computerspielen. Eine Analyse der USK-Alterseinstufungen (Forschungsbericht No. 101). Hannover: KFN.

Huston, A.C., \& Wright, J. C. (1998). Mass media and children's development. In I. E. Sigel \& K. A. Renninger (Eds.), Handbook of Child Psychology. Vol. 4: Child psychology in practice (pp. 999-1058). New York: Wiley.

Imbusch, P. (2002). Der Gewaltbegriff. In W. Heitmeyer \& J. Hagan (Eds.), Internationales Handbuch der Gewaltforschung (pp. 26-57). Wiesbaden: Westdeutscher Verlag.

Ip, Barry, Jacobs, Gabriel, \& Watkins, Alan (2008). Gaming frequency and academic performance. Australasian Journal of Educational Technology, 24(4), 355-373.

Ivarsson, M., Anderson, M., Åkerstedt, T., \& Lindblad, F. (2009 a). Playing a violent television game affects heart rate variability. Acta Pcediatrica, 98(1), 166-172.

Ivarsson, M., Anderson, M., Åkerstedt, T., \& Lindblad, F. (2009 b). Playing a violent television game does not affect saliva cortisol. Acta Pcediatrica, 98(6), 1052-1053.

Izquierdo, I., \& Medina, J. H. (1997). Memory formation: The sequence of biochemical events in the hippocampus and its connection to activity in other brain structures. Neurobiology of Learning and Memory, 68(3), 285-316.

Johnson, B. R. (1980). General occurence of stressful reactions to commercial motion pictures and elements in films subjectively identified as stressors. Psychological reports, $47,775-786$. 
Johnson, J. G., Cohen, P., Kasen, S., \& Brook, J. S. (2007). Extensive television viewing and the development of attention and learning difficulties during adolescence. Archives of Pediatrics \& Adolescent Medicine, 161(5), 480-486.

Kandel, E. R., Kupfermann, I., \& Iversen, S. (2000). Learning and Memory. In E. R. Kandel, J. H. Schwartz \& T. M. Jessell (Eds.), Principles of neural science (Vol. 4): The McGrawHill Companies.

Kirschbaum, C., Pirke, K. M., \& Hellhammer, D. H. (1993). The 'Trier Social Stress Test' - a tool for investigating psychobiological stress responses in a laboratory setting. $\mathrm{Neu}$ ropsychobiology, 28, 76-81.

Kirschbaum, C., Prüssner, J. C., Stone, A. A., Federenko, I., Gaab, J., Lintz, D., et al. (1995). Persistent high cortisol responses to repeated psychological stress in a subpopulation of healthy men. Psychosomatic Medicine, 57, 468-474.

Kirschbaum, C., Wolf, O. T., May, M., Wippich, W., \& Hellhammer, D. H. (1996). Stressand treatment-induced elavations of cortisol levels associated with impaired declarative memory in healthy adults. Life Sciences, 58(17), 1475-1483.

Kirsh, S. J. (1998). Seeing the world through mortal kombat-colored glasses: Violent video games and the development of a short-term hostile attribution bias. Childhood, 5, 177-184.

Kirsh, S. J., \& Mounts, J. R. (2007). Violent video game play impacts facial emotion recognition. Aggressive Behavior, 33(4), 353-358.

Kleimann, M., \& Mößle, T (2008). The Logs of Eliza and Other Media Stories. Behavioral and Developmental Effects of a School Based Media Education Program - Berlin Longitudinal Study Media. International Journal of Behavioral Development, 32(6), Supplement 2 (54), 55-59.

Klimmt, C. (2004). Die Nutzung von Computer- und Videospielen - Aktives Spielen am Bildschirm. In P. Rössler, H. Scherer \& D. Schlütz (Eds.), Nutzung von Medienspielen - Spiele der Mediennutzer (pp. 135-165). München: R. Fischer.

Klimmt, C. (2006). Computerspielen als Handlung - Dimensionen und Determinanten des Erlebens interaktiver Unterhaltungsangebote. Köln: Halem.

Klimmt, C., Hartmann, T., \& Frey, A. (2007). Effectance and Control as Determinants of Video Game Enjoyment. CyberPsychology \& Behavior, 10(6), 845-847.

Klimmt, C., Hartmann, T., \& Vorderer, P. (2005). Experimental studies on computer games: Is interactivity a problem? Paper presented at the ICA Conference, New York.

Klimmt, C., \& Trepte, S. (2003). Theoretisch-methodische Desiderata der medienpsychologischen Forschung über die aggressionsfördernde Wirkung gewalthaltiger Computerund Videospiele. Zeitschrift für Medienpsychologie, 15(4), 114-121.

Klimmt, C., Vorderer, P., \& Ritterfeld, U. (2004). Experimentelle Medienforschung mit interaktiven Stimuli: Zum Umgang mit Wechselwirkungen zwischen "Reiz" und "Reaktion“. In W. Wirth, E. Lauf \& A. Fahr (Eds.), Forschunglogik und-design in der Kommunikationswissenschaft (Vol. 1, pp. 142-156). Köln: von Halem.

Knecht, S., Breitenstein, C., Bushuven, S., Wailke, S., Kamping, S., Flöel, A., et al. (2004). Levodopa: Faster and better word learning in normal humans. Annals of Neurology, 56(1), 20-26.

Köller, Olaf, \& Baumert, Jürgen (2008). Entwicklung schulischer Leistungen. In Rolf Oerter \& Leo Montada (Eds.), Entwicklungspsychologie: Ein Lehrbuch (6., vollständig überarbeitete ed.). Weinheim: Beltz PVU. 
Kollmannsperger, E., Bardeleben, H., \& Bühler, K.-E. (1999). Biographischer Fragebogen (BIFA). Würzburg: Universität.

Koolstra, C. M., \& Van der Voort, T. H. A. (1996). Longitudinal effects of television on children's leisure time reading: A test of three explanatory models. Human Communication Research, 23, 4-35.

Koolstra, C. M., van der Voort, T. H. A., \& van der Kamp, L. J. T. (1997). Television's impact on children's reading comprehension and decoding skills: A 3-year panel study. Reading Research Quarterly, 32, 128-152.

Kraam-Aulenbach, N. (2000). Forschungsschwerpunkt "Virtuelle Welten". Problemlöseprozesse im Computerspiel (Forschungsbericht). Köln: FH Köln, Fachbereich Sozialpädagogik.

Kuhlmann, S., Piel, M., \& Wolf, O. T. (2005). Impaired memory retrieval after psychosocial stress in healthy young men. J. Neurosci, 25, 2977-2982.

Kunczik, M., \& Zipfel, A. (2004). Medien und Gewalt. Osnabrück: Bundesministerium für Familie, Senioren, Frauen und Jugend.

Kunter, M., Schümer, G., Artelt, C., Baumert, J., Klieme, E., Neubrand, M., et al. (2002). PISA 2000: Dokumentation der Erhebungsinstrumente. Berlin: Max-Planck-Institut für Bildungsforschung.

Lang, A., Newhagen, J., \& Reeves, B. (1996). Negative Video as Structure: Emotion, Attention, Capacity, and Memory. Journal of Broadcasting \& Electronic Media, 40(4), 460-477.

Lin, S., \& Lepper, M. R. (1987). Correlates of children's useage of video games and computers. Journal of applied social psychology, 17, 72-93.

Lupien, S. J., Gaudreau, S., Tchiteya, B. M., Maheu, F., Sharma, S., Nair, N. P. V., et al. (1997). Stress-induced declarative memory impairment in healthy elderly subjects: Relationship to cortisol reactivity. Journal of Clinical Endocrinology and Metabolism, 82(7), 2070-2075.

Lupien, S. J., \& Lepage, M. (2001). Stress, memory, and the hippocampus: can't live with it, can't live without it. Behavioural Brain Research, 127, 137-158.

Lupien, S. J., Maheu, F., Tu, M., Fiocco, A., \& Schramek, T. E. (2007). The effects of stress and stress hormones on human cognition: Implications for the field of brain and cognition. Brain and Cognition, 65, 209-237.

Maass, A., Klöpper, K-M., Michel, F., \& Lohaus, A. (2009). Effects of violent and nonviolent video games and films on cognitive processes. Paper presented at the European Conference on Developmental Psychology, Vilnius (Litauen).

Maass, A., Lohaus, A., \& Wolf, O. (2010). Media and stress in adolescent boys in Germany - Psychophysiological effects of violent and non-violent television programs and video games. Journal of Children and Media (im Druck).

Mangold, R., Winterhoff-Spurk, P., Stoll, M., \& Hamann, G. F. (1998). Veränderung des zerebralen Blutflusses bei der Rezeption emotionalisierender Filmausschnitte. Medienpsychologie, 1, 51-72.

Mason, J. W. (1968). A review of psychoendocrine research on the pituitary-adrenal cortical system. Psychosom Med, 30(5), Suppl:576-607.

Matthews, K. A., Zhu, S., Tucker, D. C., \& Whooley, M. A. (2006). Blood pressure reactivity to psychological stress and coronary calcification in the coronary artery risk development in young adults study. Hypertension, 47(3), 391-395. 
Mayrhofer, S. (2004). Der Revidierte Konzentrations-Leistungs-Test (KLT-R). In G. Büttner \& L. Schmidt-Atzert (Eds.), Diagnostik von Konzentration und Aufmerksamkeit. Tests und Trends. (Vol. 3, pp. 119-131). Göttingen: Hogrefe.

McEwen, B. S. (2000). The neurobiology of stress: from serendipity to clinical relevance. Brain Research, 886, 172-189.

Möller, I. (2006). Mediengewalt und Aggression: eine längsschnittliche Betrachtung des Zusammenhangs am Beispiel des Konsums gewalthaltiger Bildschirmspiele. Universität Potsdam, Potsdam.

Mößle, T., Kleimann, M., \& Rehbein, F. (2007). Bildschirmmedien im Alltag von Kindern und Jugendlichen: Problematische Mediennutzungsmuster und ihr Zusammenhang mit Schulleistungen und Aggressivität (1. Aufl.). Baden-Baden: Nomos.

Mößle, T., Kleimann, M., Rehbein, F., \& Pfeiffer, C. (2010). Media Use and School Achievement - Boys at Risk? British Journal of Developmental Psychology, im Druck. Retrieved from http://bpsoc.publisher.ingentaconnect.com/content/bpsoc/bjdp/pre-prints/ pjdp550

Murray, J. P., Liotti, M., Ingmundson, P. T., Mayberg, H. S., Pu, Y., Zamarripa, F., et al. (2006). Children's brain activations while viewing televised violence revealed by fMRI. Media psychology, 8, 25-37.

Myrtek, M. (2003). Fernsehkonsum bei Schülern: Ambulante psychophysiologische Untersuchungen im Alltag. Nervenheilkunde, 22, 454-458.

Myrtek, M., \& Scharff, C. (2000). Fernsehen, Schule und Verhalten: Untersuchung zur emotionalen Beanspruchung von Schülern (1. Aufl ed.). Bern u.a.: Huber.

National Television Violence Study 1 (1997). Thousand Oaks: Sage Publ.

National Television Violence Study 3 (1998). Thousand Oaks: Sage Publ.

Neuman, Susan B. (1988). The Displacement Effect: Assessing the Relation between Television Viewing and Reading Performance. Reading Research Quarterly, 23(4), 414-440.

Newhagen, J., \& Reeves, B. (1992). This evening's bad news: Effects of compelling negative television news images on memory. Journal of Communication, 42, 25-41.

OECD (2006). Are students ready for a technology-rich world? What PISA studies tell us. Paris: OECD.

OECD (Ed.). (2001). Knowledge and skills for life: First results from the OECD Programme for International Student Assessment (PISA) 2000. Paris: OECD.

Oerter, Rolf, \& Montada, Leo (Eds.). (2008). Entwicklungspsychologie: Ein Lehrbuch (6., vollständig überarbeitete ed.). Weinheim: Beltz PVU.

Owens, J., Maxim, R., McGuinn, M., Nobile, C., Msall, M., \& Alario, A. (1999). Televisionviewing habits and sleep disturbance in school children. Pediatrics, 104(3), 1-8.

Panee, C. D., \& Ballard, M. E. (2002). High versus low aggressive priming during videogame training: effects on violent action during game play, hostility, heart rate, and blood pressure. Journal of Applied Social Psychology, 32(12), 2458-2474.

Parkin, A. J. (2000). Erinnern und Vergessen (Vol. 1). Bern: Hans Huber.

Payne, J. D., \& Nadel, L. (2004). Sleep, dreams, and memory consolidation: The role of the stress hormone cortisol. Learning and Memory, 11(6), 671-678.

Pfeiffer, C., Mößle, T., Kleimann, M., Rehbein, F., Dworschak, B., Mecklenburg, E., et al. (2006). Zwischenbericht Projekt "Medienverwahrlosung als Ursache von Schulversagen". Hannover: Kriminologisches Forschungsinstitut Niedersachsen. 
Posner, J., Russell, J. A., \& Peterson, B. S. (2005). The circumplex model of affect: an integrative approach to affective neuroscience, cognitive development, and psychopathology. Dev Psychopathol, 17(3), 715-734.

Rabold, S., Baier, D., \& Pfeiffer, C. (2008). Jugendgewalt und Jugenddelinquenz in Hannover (No. 105). Hannover: Kriminologisches Forschungsinstitut Niedersachsen.

Ramm, Gesa, Prenzel, Manfred, Baumert, Jürgen, Blum, Werner, Lehmann, Rainer, Leutner, Detlev, et al. (Eds.). (2006). PISA 2003. Dokumentation der Erhebungsinstrumente. Münster: Waxmann.

Rasch, B., Friese, M., Hofmann, W., \& Naumann, E. (2010 a). Quantitative Methoden Band 1: Einführung in die Statistik für Psychologen und Sozialwissenschaftler (Vol. 3). Berlin, Heidelberg: Springer.

Rasch, B., Friese, M., Hofmann, W., \& Naumann, E. (2010 b). Quantitative Methoden Band 2: Einführung in die Statistik für Psychologen und Sozialwissenschaftler (Vol. 3). Berlin, Heidelberg: Springer.

Ravaja, N., Saari, T., Laarni, J., Kallinen, K., \& Salminen, M. (2005). The psychophysiology of video gaming: phasic emotional responses to game events Changing Views: Worlds in Play (pp. 13). Vancouver: University of Vancouver.

Ravaja, N., Turpeinen, M., Saari, T., Puttonen, S., \& Keltikangas-Jarvinen, L. (2008). The psychophysiology of James Bond: phasic emotional responses to violent video game events. Emotion, 8(1), 114-120.

Razel, Micha (2001). The Complex Model of Television Viewing and Educational Achievement. The Journal of Educational Research, 94(6), 371-379.

Rehbein, F., Kleimann, M., \& Mößle, T. (2008). Zur Einstufung des Videospiels GTA-IV durch die USK mit "Keine Jugendfreigabe". Jugendmedienschutz-Report, 3, 2-6.

Rehbein, F., Kleimann, M., \& Mößle, T. (2009 a). Computerspielabhängigkeit im Kindesund Jugendalter: Empirische Befunde zu Ursachen, Diagnostik und Komorbiditäten unter besonderer Berücksichtigung spielimmanenter Abhängigkeitsmerkmale (No. 108). Hannover: Kriminologisches Forschungsinstitut Niedersachsen.

Rehbein, F., Kleimann, M., \& Mößle, T. (2009 b). Exzessives Computerspielen und Computerspielabhängigkeit im Jugendalter: Ergebnisse einer deutschlandweiten Repräsentativbefragung. Die Psychiatrie, 6, 140-146.

Reinecke, L., \& Trepte, S. (2008). In a working mood? The effects of mood management processes on subsequent cognitive performance. Journal of Media Psychology, 20(1), 3-14.

Roberts, D. F., Foehr, U. G., \& Rideout, V. (2005). Generation M: Media in the lives of 8-18 year olds Retrieved 18.04.05], from http://www.kff.org/entmedia/entmedia030905pkg.cfm

Roe, K., Eggermont, S., \& Minnebo, J. (2001). Media use and academic achievement: Which effects? The European Journal of Communication, 26(1), 39 - 56.

Rosser, J. C., Lynch, P. J., Cuddihy, L., Gentile, D. A., Klonsky, J., \& Merrell, R. (2007). The Impact of Video Games on Training Surgeons in the 21st Century. Arch Surg, 142(2), 181-186.

Roth, G. (2001). Fühlen, Denken, Handeln: Wie das Gehirn unser Verhalten steuert (1. Aufl., Nachdr. ed.). Frankfurt am Main: Suhrkamp.

Rudolf, M., \& Müller, J. (2004). Multivariate Verfahren: Eine praxisorientierte Einführung mit Anwendungsbeispielen in SPSS. Göttingen, Bern, Toronto, Seattle: Hogrefe. 
Russell, J. A. (1980). A Circumplex Model of Affect. Journal of Personality and Social Psychology, 39(6), 1161-1178.

Salminen, M., \& Ravaja, N. (2008). Increased oscillatory theta activation evoked by violent digital game events. Neuroscience letters, 435(1), 69-72.

Sarris, V., \& Reiß, S. (2005). Ein kurzer Leitfaden der Experimentalpsychologie. München, Boston, San Fransisco, Harlow, u.a.: Pearson Studium.

Scheich, H. (2006). Visuelle Medien und unreife Gehirne. In K. Meisel \& C. Schiersmann (Eds.), Zukunft Weiterbildung. Standortbestimmungen für Forschung, Praxis und Politik. Ekkehard Nuissl von Rein zum 60. Geburtstag (pp. 223-230). Bielefeld: Bertelsmann.

Schelling, D., \& Schächtele, B. (2001). Visueller und Verbaler Merkfähigkeitstest (VVM). Frankfurt am Main: Swets Test Services.

Schlickum, M., Hedman, L., Enochsson, L., Kjellin, A., \& Felländer-Tsai, L. (2009). Systematic Video Game Training in Surgical Novices Improves Performance in Virtual Reality Endoscopic Surgical Simulators: A Prospective Randomized Study. World Journal of Surgery.

Schmidt-Atzert, L., Büttner, G., \& Bühner, M. (2004). Theoretische Aspekte von Aufmerksamkeit-/Konzentrationsdiagnostik. In G. Büttner \& L. Schmidt-Atzert (Eds.), Diagnostik von Konzentration und Aufmerksamkeit. Tests und Trends. (Vol. 3). Göttingen: Hogrefe.

Schmidt-Atzert, L., Bühner, M., \& Enders, P. (2006). Messen Konzentrationstests Konzentration? Eine Analyse der Komponenten von Konzentrationsleistungen. Diagnostica, 52(1), 33-44.

Schmidt-Atzert, L., Krumm, S., \& Bühner, M. (2008). Aufmerksamkeitsdiagnostik - Ableitung eines Strukturmodells und systematische Einordnung von Tests. Zeitschrift für Neuropsych, 19(2), 59-82.

Schmitt, M. (2004). Persönlichkeitspsychologische Grundlagen. In R. Mangold, P. Vorderer \& G. Bente (Eds.), Lehrbuch der Medienpsychologie (pp. 151-173). Göttingen u.a.: Hogrefe.

Schneider, E. F., Lang, A., Shin, M., \& Bradley, S. D. (2004). Death with a Story. How story impacts emotional, motivational, and physiological responses to first-person shooter video games. Human Communication Research, 30(3), 361-375.

Schommer, N. C., Hellhammer, D. H., \& Kirschbaum, C. (2003). Dissociation between reactivity of the hypothalamus-pituitary-adrenal axis and the sympathetic-adrenal-medullary system to repeated psychosocial stress. Psychosom Med, 65(3), 450-460.

Schrader, F.-W., \& Helmke, A. (2008). Determinanten der Schulleistung. In M. K. W Schweer (Ed.), Lehrer-Schüler-Interaktion. Inhaltsfelder, Forschungsperspektiven und methodische Zugänge (Vol. 2., pp. 285-302). Wiesbaden: VS Verlag.

Schumacher, J., Klaiberg, A., \& Brähler, E. (2001). Bevölkerungsrepräsentative Normierung der Skala zur Allgemeinen Selbstwirksamkeitserwartung. DIAGNOSTIKA, submitted.

Schwarzer, R. (1993). Streß, Angst und Handlungsregulation (Vol. 3). Stuttgart: Kohlhammer.

Schwarzer, R., \& Jerusalem, M. (Eds.). (1999). Skalen zur Erfassung von Lehrer- und Schülermerkmalen. Dokumentation der psychometrischen Verfahren im Rahmen der Wissenschaftlichen Begleitung des Modellversuchs Selbstwirksame Schulen. Berlin: Freie Universität Berlin. 
Schwenkmezger, P., Hodapp, V., \& Spielberger, C. D (1992). Das State-Trait-Ärgerausdrucks-Inventar STAXI (Vol. 1). Bern, Göttingen, Toronto: Verlag Hans Huber.

Sharif, I., \& Sargent, J. D. (2006). Association between television, movie, and video game exposure and school performance. Pediatrics, 118(4), 1061-1070.

Shin, N. (2004). Exploring pathways from television viewing to academic achievement in school age children. Journal of Genetic Psychology, 165(4), 367-381.

Singer, D. G., \& Singer, J. L. (2001). The popular media as educators and socializers of growing children. In D. G. Singer \& J. L. Singer (Eds.), Handbook of children and the media (pp. 1-6). Thousand Oaks, CA: Sage.

Skosnik, P. D., Chatterton, R. T., Swisher, T., \& Park, S. (2000). Modulation of attentional inhibition by norepinephrine and cortisol after psychological stress. International Journal of Psychophysiology, 36, 59-68.

Smith, Stacy L., Lachlan, Ken, \& Tamborini, Ron (2003). Popular Video Games: Quantifying the presentation of violence and its context. Journal of Broadcasting \& Electronic Media, 47(1), 58-76.

Spence, I., Yu, J. J., Feng, J., \& Marshman, J. (2009). Women Match Men When Learning a Spatial Skill. Journal of Experimental Psychology: Learning, Memory, and Cognition, 35(4), 1097-1103.

Spitzer, M. (2005). Vorsicht Bildschirm! Elektronische Medien, Gehirnentwicklung, Gesundheit und Gesellschaft (2. Aufl.). Stuttgart: Klett.

Staude-Müller, Frithjof, Bliesener, Thomas, \& Luthman, Stefanie (2008). Hostile and Hardened? An Experimental Study on (De-)Sensitization to Violence and Suffering Through Playing Video Games. Swiss Journal of Psychology, 67(1), 41-50.

Stevens, T., \& Mulsow, M. (2006). There is no meaningful relationship between television exposure and symptoms of attention-deficit/hyperactivity disorder. Pediatrics, 117(3), $665-672$.

Stickgold, R., Malia, A., Maguire, D., Roddenberry, D., \& O'Connor, M. (2000). Replaying the game: Hypnagogic images in normals and amnesics. Science, 290(5490), 350-353.

Subrahmanyam, K., Greenfield, P., Kraut, R., \& Gross, E. (2001). The impact of computer use on children's and adolescents' development. Journal of Applied Developmental Psychology, 22(1), 7-30.

Subrahmanyam, K., \& Greenfield, P. M. (1994). Effect of video game practice on spatial skills in girls and boys. Journal of Applied Developmental Psychology, 15, 13-32.

Subrahmanyam, K., Kraut, R., Greenfield, P., \& Gross, E. (2001). New forms of electronic media: The impact of interactive games and the internet on cognition, socialization, and behavior. In D. G. Singer \& J. L. Singer (Eds.), Handbook of children and the media (pp. 73-99). Thousand Oaks: Sage.

Tabachnick, B. G., \& Fidell, L. S. (2007). Using Multivariate Statistics (Vol. 5). Boston: Pearson.

Tazawa, Y., \& Okada, K. (2001). Physical signs associated with excessive television-game playing and sleep deprivation. Pediatrics International, 43, 647-650.

Thompson, Kimberly M., \& Haninger, Kevin (2001). Violence in E-Rated Video Games. Journal of the American Medical Association, 268(5), 591-598.

Towse, J., \& Cowan, N. (2005). Working memory and its relevance for cognitive development. In W. Schneider, R. Schumann-Hengsteler \& B. Sodian (Eds.), Young children's cognitive development. Mahwah, NJ [u.a.]: Lawrence Erlbaum Assoc. 
Trudewind, C., \& Steckel, R. (2003). Effekte gewaltorientierter Computerspiele bei Kindern: Wirkmechanismen, Moderatoren und Entwicklungsfolgen. Zeitschrift für Familienforschung, 15(3), 238-271.

van den Bulck, J. (2004 a). Media Use and Dreaming: The relationship among television viewing, computer game play, and nightmares or pleasant dreams. Dreaming, 14(1), 43-49.

van den Bulck, J. (2004 b). Television viewing, computer game playing, and internet use and self-reported time to bed and time out of bed in secondary-school children. Sleep, 27(1), 101-104.

van der Molen, J. H. W., \& Bushman, B. J. (2008). Children's direct fright and worry reactions to violence in fiction and news television. The Journal of Pediatrics(September), 420-424.

van Schie, E. G. M., \& Wiegman, O. (1997). Children and videogames: Leisure activities, aggression, social integration, and school performance. Journal of Applied Social Psychology, 27(13), 1175-1194.

Wang, M. C., Haertel, G. D., \& Walberg, H. J. (1993). Toward a knowledge base for school learning. Review of Educational Research, 63(3), 249-294.

Weinert, F. E. (2002). Perspektiven der Schulleistungsmessung - mehrperspektivisch betrachtet. In F. E. Weinert (Ed.), Leistungsmessungen in Schulen (Vol. 2, pp. 353-365). Weinheim, Basel: Beltz.

Williams, P. A., Haertel, E. D., Haertel, G. D., \& Walberg, H. J. (1982). The impact of leisuretime television on school learning: A research synthesis. American Educational Research Journal, 19, 19-50.

Willoughby, Teena (2008). A Short-Term Longitudinal Study of Internet and Computer Game Use by Adolescent Boys and Girls. Developmental Psychology, 44(1), 195-204.

Wilmers, N., Enzmann, D., Schaefer, D., Herbers, K., Greve, W., \& Wetzels, P. (2002). Jugendliche in Deutschland zur Jahrtausendwende: Gefährlich oder gefährdet? Ergebnisse wiederholter, repräsentativer Dunkelfelduntersuchungen zu Gewalt und Kriminalität im Leben junger Menschen 1998-2000 (Vol. 1). Baden-Baden: Nomos.

Wilson, B.J., Smith, S.L., Potter, W.J., Kunkel, D., Linz, D., Colvin, C.M., et al. (2002). Violence in Children's Television Programming: Assessing the Risks. Journal of Communication, 52, 5-35.

Winter, B., Breitenstein, C., Mooren, F. C., Voelker, K., Fobker, M., Lechtermann, A., et al. (2007). High impact running improves learning. Neurobiol Learn Mem, 87(4), 597-609.

Wittwer, Jörg, \& Senkbeil, Martin (2008). Is students' computer use at home related to their mathematical performance at school? Computers \& Education, 50(4), 1558-1571.

Wolf, O. T. (2009). Stress and memory in humans: Twelve years of progress? Brain Research, 1293, 142-154.

Wolf, O. T., Convit, A., McHugh, P. F., E., Kandil., Thorn, E. L., De Santi, S., et al. (2001). Cortisol differentially affects memory in young and eldery men. Behav. Neurosci., 105, 1002-1011.

Yerkes, R. M., \& Dodson, J. D. (1908). The relation of strength of stimulus to rapidity of habit-formation. Journal of Comparative Neurology and Psychology, 18(5), 459-482.

Zillmann, D. (2003). Emotionspsychologische Grundlagen. In R. Mangold, P. Vorderer \& G. Bente (Eds.), Lehrbuch Medienpsychologie (pp. 101-129). Göttingen: Hogrefe.

Zimbardo, P. G., \& Gerrig, R. J. (1999). Psychologie (Vol. 7): Springer. 
Zimmerman, F. J., \& Christakis, D. A. (2005). Children`s television viewing and cognitive outcomes: A longitudinal analysis of national data. Archives of Pediatrics \& Adolescent Medicine, 159(7), 619-625.

Zimmerman, F. J., \& Christakis, D. A. (2007). Associations between content types of early media exposure and subsequent attentional problems. Pediatrics, 120(5), 986-992. 
4. Anhang

Angang A: Studien zum Zusammenhang von Fernsehnutzung und Schulleistung

Angang B: Studien zum Zusammenhang von Computerspielnutzung und Schulleistung

Anhang C: Anwerbeflyer

Anhang D: Musteranschreiben Versuchspersonen

Anhang E: Bildschirmfotos der medialen Gewaltbedingungen

Anhang F: Versuchsleiterprotokoll

Anhang G: Versuchsleitermanual (Tag 1)

Anhang H: Versuchsassistentenmanual (Tag 1)

Anhang I: Versuchsleitermanual (Tag 2)

Anhang J: Post-Hoc-Poweranalysen

Anhang K: Explorative Streudiagramme (Distress und Kognition) 


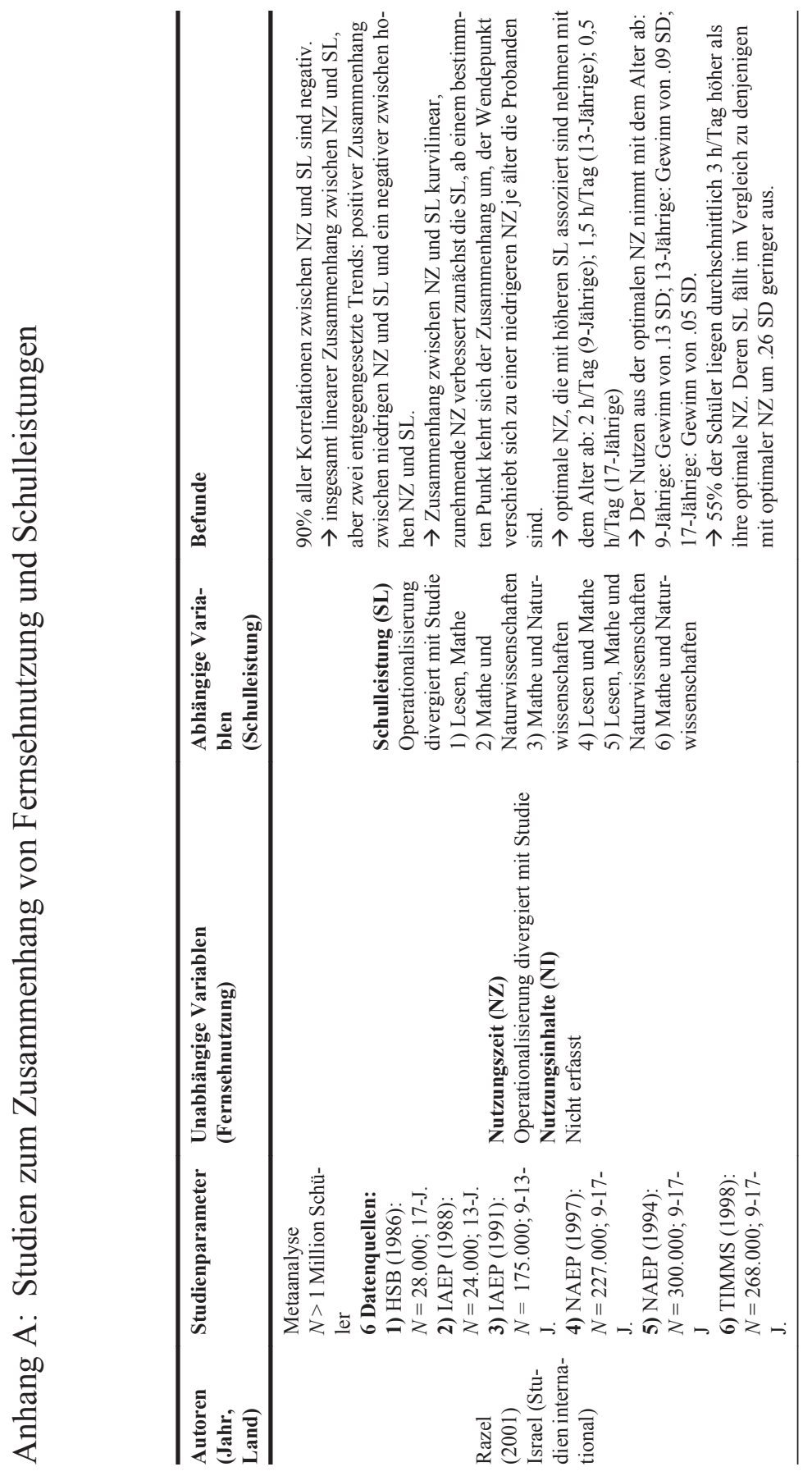

